\title{
Estimation of Tissue Contractility from Cardiac Cine-MRI Using a Biomechanical Heart Model
}

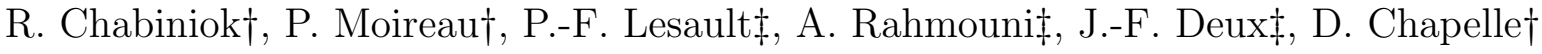 \\ $\dagger$ INRIA, Rocquencourt, B.P. 105, 78153 Le Chesnay, France \\ $\ddagger$ AP-HP Hôpital Henri Mondor, Université Paris-Est Créteil, France
}

Biomechanics and Modeling in Mechanobiology, in press

\begin{abstract}
The objective of this paper is to propose and assess an estimation procedure - based on data assimilation principles - well-suited to obtain some regional values of key biophysical parameters in a beating heart model, using actual Cine-MR images. The motivation is twofold: (1) to provide an automatic tool for personalizing the characteristics of a cardiac model in order to achieve predictivity in patient-specific modeling, and (2) to obtain some useful information for diagnosis purposes in the estimated quantities themselves. In order to assess the global methodology we specifically devised an animal experiment in which a controlled infarct was produced and data acquired before and after infarction, with an estimation of regional tissue contractility - a key parameter directly affected by the pathology - performed for every measured stage. After performing a preliminary assessment of our proposed methodology using synthetic data, we then demonstrate a full-scale application by first estimating contractility values associated with 6 regions based on the AHA subdivision, before running a more detailed estimation using the actual AHA segments. The estimation results are assessed by comparison with the medical knowledge of the specific infarct, and with late enhancement MR images. We discuss their accuracy at the various subdivision levels, in the light of the inherent modeling limitations and of the intrinsic information contents featured in the data.
\end{abstract}

\section{Introduction}

Building adequate patient-specific models is now widely recognized as a most important challenge for bioengineering applied in medicine [23], and this holds in particular for cardiac modeling $[16,46]$. Of course, as a first major ingredient, this requires the construction of accurate anatomical models which suitably represent the actual geometrical attributes of the patient considered, usually based on medical imaging data [38]. The next - at least equally important, and most challenging - stage consists in "personalizing" the biophysical characteristics of a cardiac model in order to reproduce the specificities of the patient, as e.g. in case of various pathologies which are most likely to perturb localized values of constitutive parameters. This step is crucial to render the model predictive, hence to obtain some clinically-relevant quantitative information from the model simulations, both in the current state of the patient and under various scenarii of future evolutions, such as for therapy planning, e.g. to pre-operatively optimize cardiac resynchronization therapies [45] or surgical procedures.

The complexity of the heart physiology - and of the related models - is such that this biophysical personalization procedure cannot be simply performed "manually", such as by directly extracting the required quantities from the available measurements, hence some automated 
estimation procedures are much needed in this endeavor $[40,2,18]$. Moreover, as some biophysical parameters are strongly correlated with the functional state of the organ, the automatic estimation of these parameters can be envisioned as a diagnosis tool. This dual objective of (1) circumscribing the various sources of uncertainties in the system by using measurements to estimate some unknown quantities which are key to the model behavior from a physical and physiological standpoint, thus providing useful information for diagnosis, and (2) thereby rendering the biophysical model adequately predictive in subsequent simulations, is essential in this paper. This corresponds - indeed - to the concept of data assimilation already widely employed in other domains of science and engineering, such as geophysics in general and weather forecasting in particular, see e.g. [8]. We emphasize that this approach is notably different from the objective of cardiac motion tracking per se [37,31, 9, 44], sometimes complemented by extracting some valuable indices from available data by using some constraining physical model equations $[21,30]$. The discriminating criterion in data assimilation is that once the estimation has been performed the biophysical model must be able to run independently of any data, while providing accurate and predictive solutions. Hence, the data assimilation framework requires two essential components: (1) a biophysical model of well-controlled complexity in order to ensure predictivity in forward simulations without undue sources of uncertainties relative to the available data, namely, unobservable phenomena, and (2) an effective data assimilation procedure well-adapted to the model equations and to the data in question.

In this paper, we will consider a biomechanical heart model as proposed in [43] and further substantiated in [13], with some clinical validations performed in [11, 45]. For this model we will demonstrate a complete personalization procedure - namely, both anatomical and biophysical - using actual clinical data consisting of in-vivo Cine-MR image sequences and pressure measurements, based on some earlier-proposed data assimilation procedures [33, 34, 32]. In order to allow for a detailed assessment of the estimation results, we specifically devised and performed an animal experiment in which a controlled infarct was created [11], and imaging and pressure data acquired before and after infarction - at two subsequent stages. Hence, we can quantitatively compare the estimation results pertaining to regional values for a major biophysical parameter of interest in this context - namely, the tissue contractility which characterizes the ability of the muscle fiber to generate active stresses under the effect of electrical activation - at the various stages. Moreover, we can also qualitatively and semi-quantitatively evaluate the post-infarct results with respect to a priori knowledge regarding the pathology and to late enhancement indicators. We point out that we selected the Cine-MRI modality to perform this estimation trial - despite its rather limited contents through the boundary segmentation postprocessing in terms of volume-distributed physical information, compared to e.g. tagged MRI - because it is a rather standard clinical imaging protocol for this type of pathology. Therefore, while the specific modality considered - Cine-MRI - is not by far the most favorable choice for our specific estimation objectives of determining localized biophysical parameter values, our estimation procedure can be seen as a challenge deliberately oriented towards actual clinical practice.

The outline of the paper is as follows. In Section 2 we describe the clinical data collected in the animal experiment, and the associated anatomical modeling. Then, in Section 3 we present the biophysical heart model and the proposed data assimilation procedure, with some related algorithmic and computational considerations for both. Next, in Section 4 we describe the specific estimation trial performed in this study, namely, the estimation of regional tissue contractility quantities using Cine-MRI, and we report on the actual results, which are subsequently discussed and assessed in Section 5. Finally we give some concluding remarks in Section 6 . 


\section{Clinical data and anatomical modeling}

\subsection{Experimental protocol}

The experimental data used in this article were obtained as part of an animal study approved by the Institutional Animal Care and Use Committee of the Faculty of Medicine of Université Paris-Est Créteil, France. The animal - a farm pig of $30 \mathrm{~kg}$ - was housed and cared according to European Union standards. Before each data acquisition, the animal was premedicated with intramuscular Ketamin $(15 \mathrm{mg} / \mathrm{kg})$, anesthetized with Propofol $(0.35 \mathrm{mg} / \mathrm{kg}$ intravenous bolus and $0.05 \mathrm{mg} / \mathrm{kg} / \mathrm{min}$ continuous infusion), intubated and ventilated, see [11] for the detailed experimental protocol.

Firstly, the subject was examined and data acquired once in a baseline condition (physiological heartbeat). Then, an antero-septal myocardial infarction was induced by a 120-min occlusion of the mid-part of the left anterior descending coronary artery, using a balloon catheter, followed by reflow. This occlusion led to an extended myocardial infarction, as was confirmed in the follow-up by the late enhancement MR images. The animal was re-examined 10 and 38 days after the infarct creation. These various stages are referred to as baseline, $T_{0}+10$ and $T_{0}+38$ in the sequel. The $T_{0}+10$ data acquisition corresponds to the acute stage of the infarct (edematous necrotic tissue with only slightly diminished wall thickness), while at $T_{0}+38$ we could already expect - and indeed observed - thinning of the LV wall (caused by resorption of the necrosis and tissue remodeling).

For each of the studied stages (baseline, $T_{0}+10$ and $T_{0}+38$ ), non-invasive MR image data were acquired and pressures in the heart cavities and large vessels were measured. More precisely, the image data were acquired using a Philips Achieva 3T MR system $(200 \mathrm{mT} / \mathrm{m} / \mathrm{ms}$ slew rate, maximal gradient strength $80 \mathrm{mT} / \mathrm{m}, 32$-channel phased-array cardiac coil). The following types of MR data were obtained:

- Cine in retrospective ECG gating (incoherent gradient echo, flip angle $15^{\circ}$, TR $\sim 6 \mathrm{~ms}$, TE $\sim 3.5 \mathrm{~ms}$, turbo factor 5 , SENSE 1, acq. matrix 210x160, voxel size 1.5x1.7x8 mm, no intergap between short axis slices, 40 time frames / cardiac cycle);

- Late enhancement images (gadolinium chelate contrast agent - gadoterate dimeglumine, Dotarem, $0.2 \mathrm{mmol} / \mathrm{kg}$, T1 weighted gradient echo, 3D acquisition, flip angle $15^{\circ}$, TR $\sim 5.0 \mathrm{~ms}$, TE $\sim 2.4 \mathrm{~ms}$, TI $\sim 220 \mathrm{~ms}$, turbo factor 32 , SENSE 2 , acq. matrix $175 \times 175 \times 15$, voxel size $1.5 \times 1.5 \times 5 \mathrm{~mm}$, magnitude and phase sensitive reconstructions) to visualize the infarct.

The pressures were measured invasively by means of retrograde catheterization (aortic and left ventricle pressure) and by antegrade catheterization (right atrium, right ventricle, pulmonary artery and pulmonary capillary wedged pressure).

\subsection{Anatomical Model}

Anatomical models were created for each stage based on the end-diastolic time frames of the Cine-MR images, a state considered to be close to a stress-free configuration. Hence, reference configurations have been determined from this configuration according to the inverse procedure described in [35]. Segmentations of the ventricles up to the valve plane were performed manually in this particular time frame by an expert using the CardioViz3D software [49]. Then a computational mesh was built in the segmented geometry using the Yams and GHS3D meshing tools [17], see the resulting mesh in the baseline configuration in Figure 1. Note that the atria are not contained in this anatomical model. The computational mesh typically features at least 

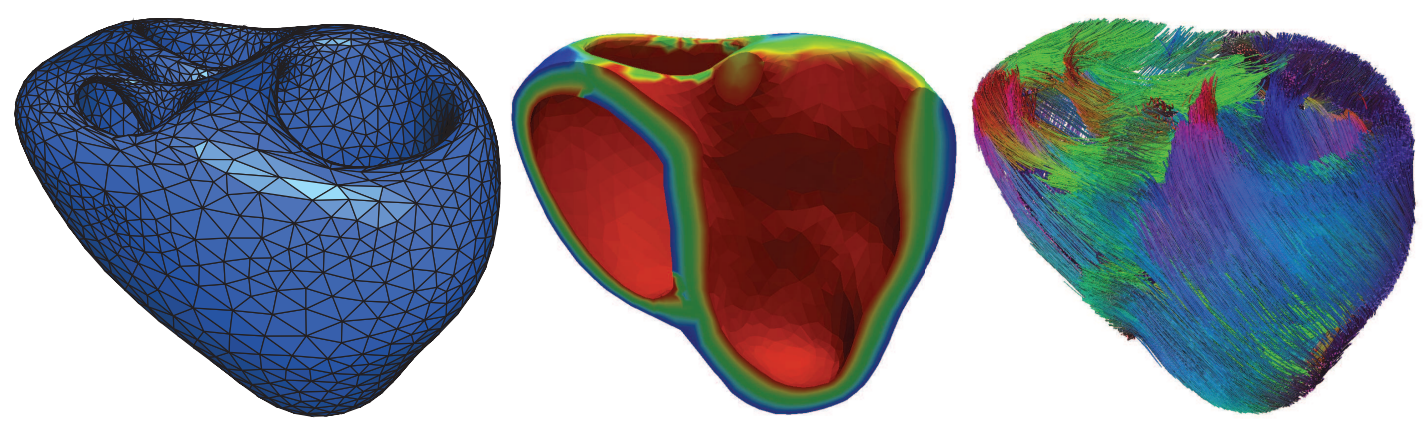

Figure 1: Computational mesh for baseline (left), distance from endocardium to epicardium map (center) and generic fibers prescribed in the mesh (right)

two elements across the thickness throughout the whole left ventricular wall, which was shown to provide reasonable accuracy - especially compared to other modeling and measurement uncertainties - in our previous validation studies [11, 45].

The fibers - needed in the biomechanical model as privileged directions of contraction (see Section 3.1) - were defined in the same anatomical model based on general anatomical knowledge [42]: $-60 / 60$ degrees elevation in the left ventricle, $-50 / 50$ in the right ventricle from epicardium to endocardium. To that purpose, we first computed a 3D distance map [4] from any point in the tissue to the epicardium and endocardium, see Figure 1. This distance and its corresponding gradient were then employed to compute the fiber elevation across the thickness. The final fiber directions for the baseline case are also shown in Figure 1, visualized with the MedINRIA software [50]. We point out that we considered variations of these angle parameters within the pre-calibration of the model - see Section 4.1 - and found that the above values provided adequate global motion patterns as regards longitudinal versus radial strains.

\subsection{Processed data}

The data assimilation procedure uses measurements - also called observations - to estimate various uncertainties in the biophysical model. These measurements consist in a time sequence of data. In this study, our purpose is to use a sequence of segmentations for the region of interest obtained from the MRI as initially presented in [34]. Therefore, we have manually segmented in the three short axis Cine-MR image sequences (baseline, $T_{0}+10, T_{0}+38$ ) the endocardium and epicardium of the left ventricle, where by LV epicardium we refer to the part of the epicardial surface adjacent to the LV together with the septal part of the RV endocardium. These two surface segmentations are transformed into two triangular meshes using CardioViz3D. We point out that at each time step the two meshes are generated independently of the previous time steps so that the number of triangles varies for the endocardium from about 4,500 to 5,500 and for the epicardium from about 5,000 to 6,000.

\section{Methods}

\subsection{Heart model}

In this section, we summarize the formulation of the heart model considered - described in more detail in $[43,13]$, which also compare this model to other formulations proposed in the 
literature, such as in $[36,22]$ - and we give the corresponding discrete equations employed in our numerical simulations.

\subsubsection{Continuum mechanics formulation}

Let $\Omega_{0}$ be a reference configuration for the system. Denoting the deformation of the solid medium by

$$
\underline{\varphi}: \begin{aligned}
\Omega_{0} \times[0, T] & \longrightarrow \Omega(t), \\
(\underline{\xi}, t) & \longmapsto \underline{x}=\underline{\varphi}(\underline{\xi}, t)
\end{aligned}
$$

the displacement of the domain is given by $\underline{y}(\underline{\xi}, t)=\underline{\varphi}(\underline{\xi}, t)-\underline{\xi}$, and the velocity of a material point $\underline{\xi}, \partial_{t} \underline{\varphi}(\underline{\xi}, t)=\partial_{t} \underline{y}(\underline{\xi}, t)$ is denoted by $\underline{v}$. We then introduce the deformation gradient

$$
\underline{\underline{F}}(\underline{\xi}, t)=\underline{\nabla}_{\underline{\xi}} \underline{\varphi}(\underline{\xi}, t),
$$

such that the deformed volume is given by $J d \Omega$ where $J=\operatorname{det} \underline{\underline{F}}$ and $d \Omega$ is the volume measure (here in the reference configuration), while a change of area is given by $J \underline{\underline{F}}^{-T} \cdot \underline{d S}$. Furthermore, we introduce the right Cauchy-Green deformation tensor $\underline{\underline{C}}=\underline{\underline{F}}^{T} \cdot \underline{\underline{F}}$, while recalling that local changes of geometry are described by the Green-Lagrange strain tensor

$$
\underline{\underline{e}}=\frac{1}{2}(\underline{\underline{C}}-\underline{\underline{\mathbb{1}}})=\frac{1}{2}\left(\underline{\underline{\nabla}}_{\underline{\xi}} \underline{y}+\left(\underline{\underline{\nabla}}_{\underline{\xi}} \underline{y}\right)^{T}+\left(\underline{\underline{\nabla}}_{\underline{\xi}} \underline{y}\right)^{T} \cdot \underline{\underline{\nabla}}_{\underline{\xi}} \underline{y}\right) .
$$

The principle of virtual work written in the reference configuration - namely in Lagrangian form - reads

$$
\forall \underline{v}^{*} \in \mathcal{V}, \quad \int_{\Omega_{0}} \rho_{0} \underline{\gamma} \cdot \underline{v}^{*} d \Omega+\int_{\Omega_{0}} \underline{\underline{\Sigma}}: \mathrm{d}_{\underline{y}} \underline{\underline{e}} \cdot \underline{v}^{*} d \Omega=\int_{\Omega_{0}} \rho_{0} \underline{f} \cdot \underline{v}^{*} d \Omega+\int_{\Gamma_{N}^{s}} \underline{t}_{0} \cdot \underline{v}^{*} d S,
$$

where $\underline{\gamma}=\partial_{t} \underline{v}(\underline{\xi}, t)$ denotes the acceleration, $\rho_{0}$ is the solid mass per unit volume, $\underline{\underline{\Sigma}}$ is the second Piola-Kirchhoff stress tensor, $f$ are volume-distributed forces, $\underline{t}_{0}$ surface-distributed forces applied on $\Gamma_{N}^{\mathrm{s}} \subset \partial \Omega_{0}$ and

$$
\mathrm{d}_{\underline{y}} \underline{\underline{e}} \cdot \underline{v}^{*}=\frac{1}{2}\left(\left(\mathrm{~d}_{\underline{y}} \underline{\underline{F}} \cdot \underline{v}^{*}\right)^{T} \cdot \underline{\underline{F}}+\underline{\underline{F}}^{T} \cdot \mathrm{d}_{\underline{y}} \underline{\underline{F}} \cdot \underline{v}^{*}\right)
$$

corresponds to the derivative of the Green-Lagrange strain tensor with respect to displacements in the admissible space of test functions $\mathcal{V}$. Concerning the inclusion of inertia effects which are not always taken into account in heart models, we point out that order of magnitude calculations indicate that they are roughly comparable to diastolic ventricular pressure loading. Furthermore, whereas any mathematical model is bound to contain errors of various sources, the inertia term per se is hardly prone to any error at all.

\subsubsection{Constitutive law}

We model the complex behavior of the cardiac tissue by combining an active constitutive law in the muscle fiber direction $\underline{\tau}_{1}$ with a visco-hyperelastic material. This mixture is depicted in Figure 2 as a rheological model describing the combination of constitutive ingredients. In order to ensure energy consistency, the series law in this schematic is more intricate than the usual linear rheological rule - namely additive strains. According to [43], this rule becomes

$$
1+2 e_{1 \mathrm{D}}=\left(1+2 e_{s}\right)\left(1+2 e_{c}\right)
$$




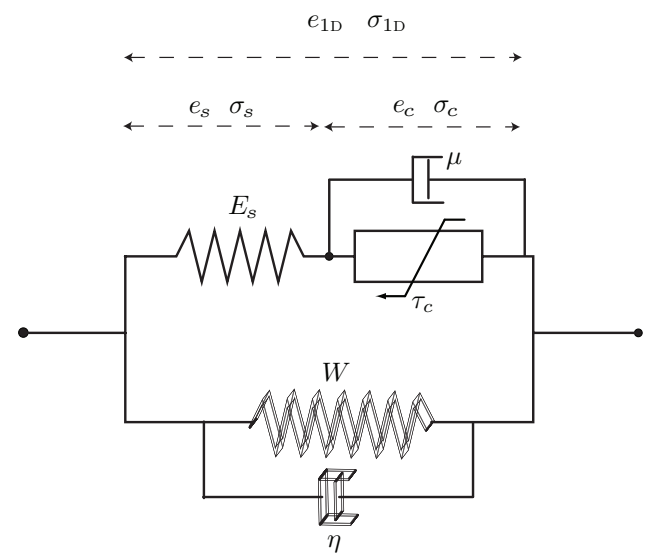

Figure 2: Hill-Maxwell rheological model - Combination of 3D passive (bottom) and 1D fiberoriented active (top) branches

with $e_{1 \mathrm{D}}=\underline{\tau}_{1} \cdot \underline{\underline{e}} \cdot \underline{\tau}_{1}$, and

$$
\sigma_{1 \mathrm{D}}=\frac{\sigma_{c}}{1+2 e_{s}}=\frac{\sigma_{s}}{1+2 e_{c}} .
$$

In addition, the parallel association in Figure 2 consists in decomposing the stress tensor into

$$
\underline{\underline{\Sigma}}=\underline{\underline{\Sigma}}_{p}+\sigma_{1 \mathrm{D}} \underline{\tau}_{1} \otimes \underline{\tau}_{1} \text {. }
$$

Passive 3D branch The rheological model already takes into account the anisotropy in the myocardial fiber direction $\underline{\tau}_{1}$ and we will then employ an isotropic hyperelastic law for the 3D passive branch. Note that in the future we could also consider anisotropic passive laws such as proposed in $[36,15,19]$ and e.g. assess the estimation of the corresponding constitutive parameters [52]. In our case, denoting by $W_{e}$ the hyperelastic potential, the corresponding stress contribution is given by $\frac{\partial W_{e}}{\partial \underline{e}}$ where - according to the Rivlin-Ericksen theorem [41] - the hyperelastic potential is necessarily a function of the so-called invariants $\left(I_{1}, I_{2}, I_{3}\right)$ of the tensor $\underline{\underline{C}}$ defined by

$$
I_{1}=\operatorname{tr} \underline{\underline{C}}, \quad I_{2}=\frac{1}{2}\left((\operatorname{tr} \underline{\underline{C}})^{2}-\operatorname{tr}\left(\underline{\underline{C}}^{2}\right)\right), \quad I_{3}=\operatorname{det}(\underline{\underline{C}})=J^{2} .
$$

When considering nearly incompressible materials as is the case for soft tissues, it is convenient (see [29]) to instead use the reduced invariants $\left(J_{1}, J_{2}, J_{3}\right)$

$$
J_{1}=I_{1} I_{3}^{-\frac{1}{3}}, \quad J_{2}=I_{2} I_{3}^{-\frac{2}{3}}, \quad J_{3}=I_{3}^{\frac{1}{2}}=J
$$

with which the stress tensor naturally decomposes into its volumic and deviatoric forms. Hence, in this paper we assume

$$
W_{e}=W_{e}\left(J_{1}, J_{2}, J_{3}\right),
$$

and in practice we employ an extension of the Ciarlet-Geymonat potential [14], namely,

$$
W_{e}=\kappa_{1}\left(J_{1}-3\right)+\kappa_{2}\left(J_{2}-3\right)+\kappa((J-1)-\ln (J)) .
$$

Soft tissues are also highly damped materials, and therefore we complement the hyperelastic behavior by introducing a viscous pseudo-potential $W_{v}$ assumed to satisfy the dissipation property

$$
\frac{\partial W_{v}}{\partial \underline{\underline{\dot{e}}}}(\underline{\underline{e}}, \underline{\underline{\dot{e}}}): \underline{\underline{\dot{e}}} \geq 0, \quad \forall(\underline{\underline{e}}, \underline{\underline{e}}) .
$$


Here we choose a simple pseudo-potential, i.e. $W_{v}=\frac{\eta}{2} \operatorname{tr}\left(\underline{\underline{\dot{e}}}^{2}\right)$, so that finally

$$
\underline{\underline{\Sigma}}_{p}=\frac{\partial W_{e}}{\partial \underline{\underline{e}}}+\frac{\partial W_{v}}{\partial \underline{\underline{\underline{e}}}}=\sum_{1 \leq i \leq 3} \frac{\partial W_{e}}{\partial J_{i}} \frac{\partial J_{i}}{\partial \underline{\underline{e}}}+\eta \underline{\underline{\dot{e}}} .
$$

Active 1D branch In the fiber direction, we combine a passive linear series element of elasticity modulus $E_{s}$, a linear damping element of coefficient $\mu$, and the active element proposed in [7] and further refined in [13]. Note in passing that the 1D elastic element in itself induces passive anisotropy for the overall model. The active element builds up on Huxley's model [24] using a multiscale approach, and can be summarized in the following active dynamical constitutive equations

$$
\left\{\begin{array}{l}
\dot{k}_{c}=-\left(|u|+\alpha\left|\dot{e}_{c}\right|\right) k_{c}+n_{0} k_{0}|u|_{+} \\
\dot{\tau}_{c}=-\left(|u|+\alpha\left|\dot{e}_{c}\right|\right) \tau_{c}+\dot{e}_{c} k_{c}+n_{0} \sigma_{0}|u|_{+}
\end{array}\right.
$$

In this system, $e_{c}$ denotes the strain of the active component - directly associated with the sarcomere units in the myofibers $-\tau_{c}$ represents the corresponding active stress arising from the actin-myosin bonds, and $k_{c}$ is an equivalent stiffness. The quantity $u$ should be seen as an input which summarizes the electrical activation, and is defined by

$$
u(t)=k_{A T P} \mathbb{1}_{\left[C a^{2+}\right] \geq C}-k_{S R} \mathbb{1}_{\left[\mathrm{Ca}^{2+}\right]<C},
$$

where $\mathbb{1}$. denotes the indicator function of the set appearing in the subscript. Hence $u$ takes two different values - corresponding to two different reaction rates $k_{A T P}$ and $k_{S R}$ - and changes sign according to the concentration of calcium ions bound on troponin-C. When this concentration is above the threshold $C, u=k_{A T P}$ is positive and induces an increase in active stiffness and stress, namely, contraction (systole). By contrast, a concentration below the threshold induces $u=-k_{S R}<0$ and relaxation (diastole). In addition, the remaining quantities in (9) denote various biophysical parameters to be prescribed. In particular, $k_{0}$ and $\sigma_{0}$ represent the maximum values of active stiffness and stress - respectively - which can be reached during systole, while $n_{0}$ is the current fraction of recruitable bridges. Hence, $n_{0}$ may fluctuate depending on various external variables related to the perfusion state - e.g. the oxygen concentration - the preload stress and strain reached in the current and/or previous cycles, etc., see [13]. In this work, we will only consider constant $n_{0}=1$.

Finally, the total stress in the active element is modeled by combining $\tau_{c}$ with a viscous contribution in the form

$$
\sigma_{c}=\tau_{c}+\mu \dot{e}_{c} .
$$

We emphasize that in the active behavior the parameter $\sigma_{0}$ - which we will now refer to as the contractility - is particularly important since it directly determines how the electrical activation is able to induce stress variations. This is why we will focus on the estimation of this specific parameter in the sequel.

\subsubsection{Circulation modeling and boundary conditions}

Circulation modeling The effect of the blood pressure in the ventricular cavity - denoted by $P_{v}$ - is represented in the principle of virtual work (1) by the variational loading term

$$
\forall \underline{v}^{*} \in \mathcal{V}, \quad \mathcal{P}_{\text {ext }}\left(\underline{v}^{*}\right)=-\int_{\Gamma_{\text {endo }}} P_{v} \underline{n} \cdot \underline{\underline{F}}^{-1} \cdot \underline{v}^{*} J d S,
$$




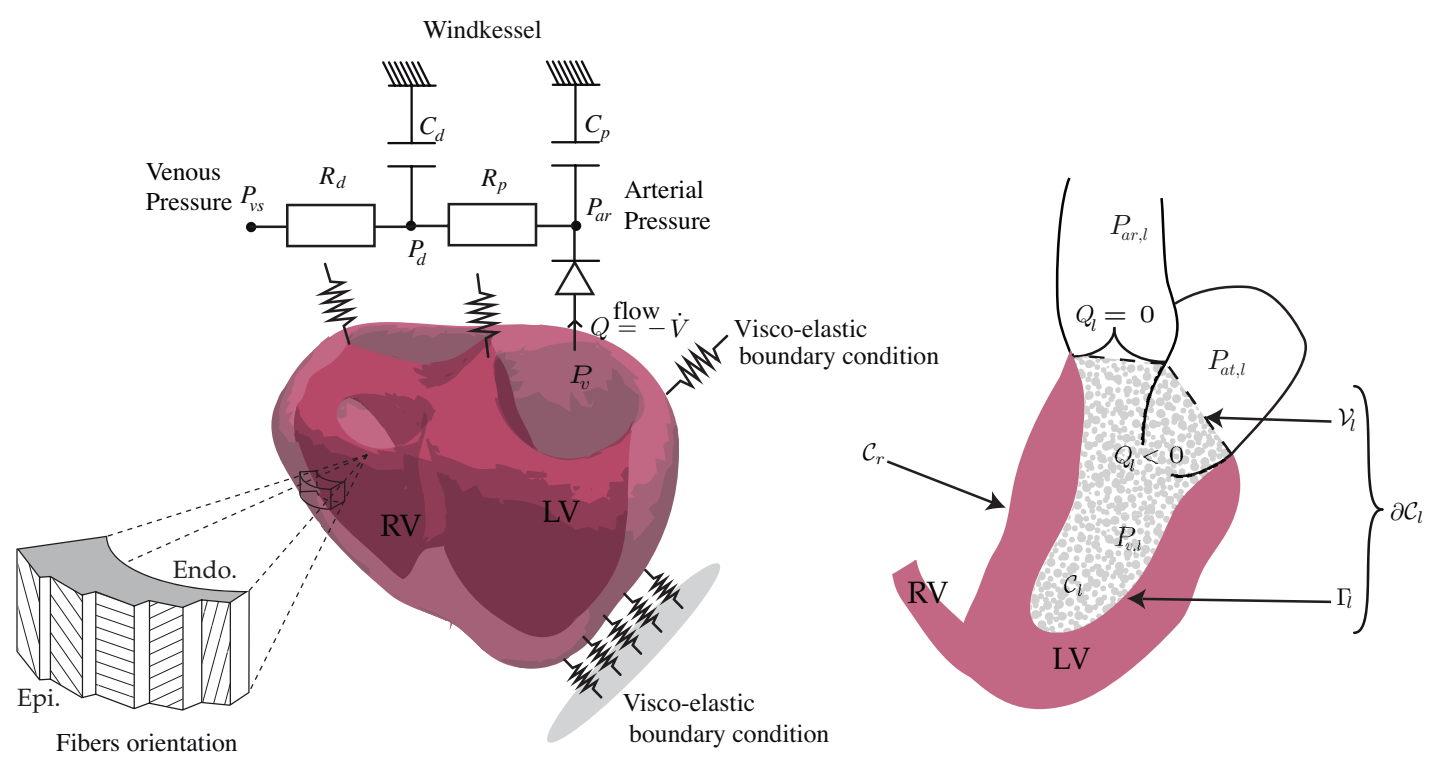

Figure 3: Anatomical model, boundary conditions and circulation model

to be incorporated in the boundary traction virtual work, where $\underline{n}$ is the endocardium outward unit normal vector in the reference configuration. When the pressure is measured, it can be directly prescribed in this expression leading to the so-called following pressure non-linear effect induced by large displacements of the cavity.

When the pressure data is not available or too noisy, we can instead determine $P_{v}$ using a simplified model of the circulatory system as explained in [43]. In this case $P_{v}$ is assumed to be homogeneous in each compartment - as a reasonable first-order approximation - and the circulation model consists in combining a Windkessel model (sometimes also called lumped parameter model) for the arterial pressure and a simplified representation of the mitral and aortic valves by analogy to diodes (see Figure 3) imposing a constant volume during isovolumic phases and pressure continuity when a valve is open. Regarding the implementation of the corresponding constraints, we adopt a penalized strategy by relating for each ventricle cavity the outward flow $Q_{c}$ - equal to the opposite of the volume change rate $\dot{V}_{c}$ - to the difference of pressures with the adjacent cavities as follows (see also Fig. 3)

$$
\begin{aligned}
\forall c=l, r, \quad Q_{c} & =-\dot{V}_{c}=-\int_{\partial \mathcal{C}_{c}} \underline{\underline{n}} \cdot \underline{\underline{F}}-\underline{\underline{v}} J d S \\
& =f_{\delta, c}\left(P_{v, c}, P_{a r, c}, P_{a t, c}\right)=\left\{\begin{array}{l}
K_{i s o, c}\left(P_{v, c}-P_{a t, c}\right) \text { if } P_{a t, c} \leq P_{v, c} \leq P_{a r, c} \\
K_{a r, c}\left(P_{v, c}-P_{a r, c}\right)+K_{i s o}^{i}\left(P_{a r, c}-P_{a t, c}\right) \text { if } P_{v, c} \geq P_{a r, c} \\
K_{a t, c}\left(P_{v, c}-P_{a t, c}\right) \text { if } P_{v, c} \leq P_{a t, c}
\end{array}\right.
\end{aligned}
$$

where $K_{a r}$ and $K_{a t}$ - associated with open valves - are taken much larger than $K_{\text {iso }}$ [43]. The atrium pressures are prescribed with physiological values at a constant low level during most of the cardiac cycle, and at a given higher level during atrial contraction. By contrast, the modeling of arterial pressures dynamics necessitates more care, hence we assume each of these pressures to be governed by a 4-element Windkessel model of two $R-C$ systems in series as 
represented in Figure 3. This gives

$$
\left\{\begin{array}{l}
Q_{c}=-\dot{V}_{c}=-\int_{\partial \mathcal{C}_{c}} \underline{\underline{n}} \cdot \underline{\underline{F}}-\underline{\underline{v}} J d S=f_{\delta, c}\left(P_{v, c}, P_{a r, c}, P_{a t, c}\right), \quad c=l, r \\
C_{p, c} \dot{P}_{a r, c}+\frac{P_{a r, c}-P_{d, c}}{R_{p, c}}=Q_{c}, \quad c=l, r \\
C_{d, c} \dot{P}_{d, c}+\frac{P_{d, c}-P_{a r, c}}{R_{p, c}}=\frac{P_{v s, c}-P_{d, c}}{R_{d, c}}
\end{array}\right.
$$

Boundary conditions The issue of adequate boundary conditions to be prescribed on the cardiac mechanical system is crucial and remains a challenge, in particular as concerns the effect of the surrounding organs. More specifically, the heart is in contact with the diaphragm, the thoracic cage, the lungs and is also partially sustained by the large vessels (aorta, pulmonary artery and veins). A visible consequence - as seen for example in a Cine-MR image sequence - of these contact and tethering conditions is that the beating heart descends into the cardiac cavity blocked by the diaphragm, so that the apex displaces very little.

It is clearly impossible to take into account in detail all the surrounding organs to define exact boundary conditions that would lead to solving multiple contact problems with complex contact conditions. Our approach is then to devise relevant simplified boundary conditions as proposed in [35] by considering viscoelastic support boundary conditions in adequate regions. From a variational standpoint, this can be written as the additional external load virtual work

$$
\forall \underline{v}^{*} \in \mathcal{V}, \quad \mathcal{P}_{\text {supp }}\left(\underline{v}^{*}\right)=-\int_{\mathcal{B}}(a(\underline{\underline{\mathrm{x}}}) \underline{y}+b(\underline{\underline{\mathrm{x}}}) \underline{v}) \cdot \underline{v}^{*} d S,
$$

on the boundary $\mathcal{B}$ with $a$ and $b$ some positive elastic and viscoelastic moduli.

Using the Cine-MRI for reference we define $\mathcal{B}$ in three different parts: (1) the upper boundary of the system considered, corresponding to the attachment with the atria, large arteries and veins, namely, the so-called base; (2) a tethering area between the apex and the thoracic cage and diaphragm; and (3) a contact area between the anterior wall and the thoracic cage.

\subsubsection{Electrical activation}

The last important ingredient in our model is the definition of the input variable $u$ which represents the electrical activation of the tissue. This variable could be described using specific electrophysiological models of various levels of complexity $[12,48]$. In the context of this study, however, the pathology is not associated with electrical activation defects, hence mainly related to the tissue mechanical response. Therefore, the details of the electrical activation patterns are of lesser consequence than the global timing of the electrical stimulation propagation. We can then prescribe the input variable using analytical expressions for $u$ in which the propagation velocity is adjusted so that the complete activation duration corresponds to the measured QRS length, with various wave shapes. We more particularly considered a wave pattern obtained by weighing a planar wave traveling from apex to base by a function representing the transmural propagation at a lower velocity from endocardium to epicardium, see Figure 4. Alternatively, we tested other shapes - such as a simple planar or spherical waves - and obtained very similar behaviors in the mechanical contractions - hence, in the estimation results - provided the wave propagation was adjusted based on the QRS duration.

\subsubsection{Discretization procedures}

The above model is solved using a finite element spatial discretization [5]. For the displacement variable, a $\mathbb{P}_{1}$-Lagrange discretization is chosen in order to use the geometrical mesh 


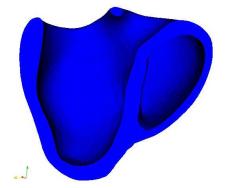

(a) $0 \mathrm{~ms}$ - enddiastole

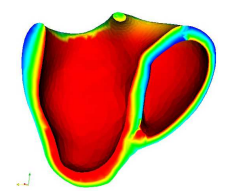

(f) $70 \mathrm{~ms}$

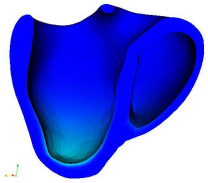

(b) $30 \mathrm{~ms}$

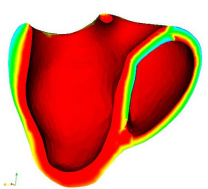

(g) $80 \mathrm{~ms}$

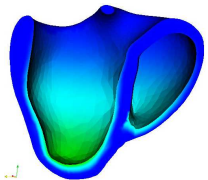

(c) $40 \mathrm{~ms}$

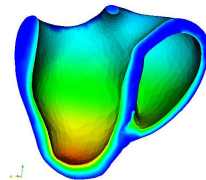

(d) $50 \mathrm{~ms}$

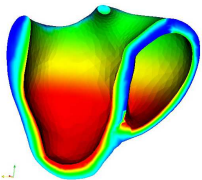

(e) $60 \mathrm{~ms}$

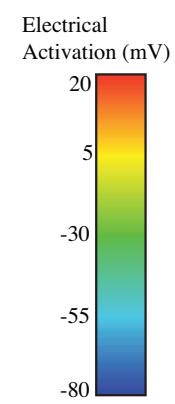

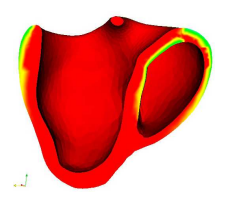

(h) $90 \mathrm{~ms}$

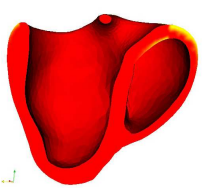

(i) $100 \mathrm{~ms}$

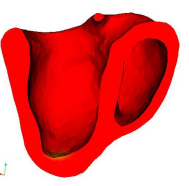

(j) $300 \mathrm{~ms}-$ end-systole

Figure 4: Analytical prescription of the electrical activation at the baseline stage: the planar wave activating the subendocardial part propagates fast from the apex to the base, together with the slower transmural propagation

generated from the segmentation. Thus we restrict the bulk modulus to values corresponding to an equivalent Poisson ratio $\nu=0.49$, for which numerical experiments show that numerical locking is not an issue, while the material is still nearly-incompressible. We denote by the vector $Y^{n}=\left\{\underline{y}_{h}^{n}\right\}$, the collection of displacement degrees of freedom associated with the $\mathbb{P}_{1}$-Lagrange solution function $\underline{y}_{h}^{n}$ at time $t_{n}$. Similarly, we introduce $V^{n}=\left\{\underline{v}_{h}^{n}\right\}$ the velocity degrees of freedom vector. Regarding the internal variables $\left(\tau_{c}, k_{c}, e_{c}\right)$, they need to be defined at the numerical integration points $\underline{P}_{i}$ in order to compute the second Piola-Kirchhoff stress tensor. We denote by $\left\{e_{c, h}^{n}\right\},\left\{\tau_{c, h}^{n}\right\}$ and $\left\{k_{c, h}^{n}\right\}$ the associated collection vectors.

We then need to define an adequate time discretization scheme, which we do with a particular concern for the preservation of energy balances, which ensures the numerical stability of the simulations [13]. This provides a relationship between the values of the discrete state variable defined by $X^{n}=\left(Y^{n} ; V^{n} ;\left\{e_{c, h}^{n}\right\} ;\left\{\tau_{c, h}^{n}\right\} ;\left\{k_{c, h}^{n}\right\}\right)$ at two subsequent time steps. We give the details of the specific time scheme devised for the above model in Appendix A. Note that, when the ventricular pressures are not available as data, the valve and Windkessel models need also be discretized and the state variable then contains the corresponding pressure values, namely, $X^{n}=\left(Y^{n} ; V^{n} ;\left\{e_{c, h}^{n}\right\} ;\left\{\tau_{c, h}^{n}\right\} ;\left\{k_{c, h}^{n}\right\} ; P_{a r, c=l, r}^{n} ; P_{d, c=l, r}^{n}\right)$.

\subsection{Data assimilation method}

In order to proceed with the presentation of adequate data assimilation methods, let us summarize and generalize the above-discussed type of dynamical model in the generic formalism

$$
\left\{\begin{array}{l}
\dot{x}=\mathcal{A}(x, \vartheta, t) \\
x(0)=x_{0}+\zeta_{x} \\
\vartheta=\vartheta_{0}+\zeta_{\vartheta}
\end{array}\right.
$$

where $x$ is the state vector characterizing the trajectory of the system in the phase space, $\mathcal{A}$ denotes a differential operator, and $\vartheta$ represents a collection of constitutive parameters. The state initial condition $x(0)$ and the parameters are each decomposed into two terms, namely, some a priori values $x_{0}$ and $\vartheta_{0}$ representative of the type of system of interest, and variations $\zeta_{x}$ and $\zeta_{\vartheta}$, which more precisely characterize - or personalize - the specific system at hand. We 
point out that with $\vartheta$ we focus only on patient-specific parameters of interest in a given context, whereas other parameter values are directly embedded into the definition of $\mathcal{A}$.

Modeling and simulating the target real system (14) consists in defining an adequate discretized model $\mathcal{A}_{h}$ acting on discretized versions $x_{h}$ and $\theta$ of the state and parameter variables, respectively, to approximate the trajectory of (14). In our case, it is the heart model and its discretization presented in Section 3.1 which we will consider in this formalism, and the state variable $x_{h}$ can be represented by a finite dimensional state vector denoted by $X$ and of possibly very large size (typically from thousands to millions of degrees of freedom). Note that in order to avoid clouding this discussion with unnecessary technical details, we only present the principles using the space semi-discretized equations, see Sections 3.2.2 and 3.2.3 for the specific developments of our approach with complete time-space discretization. By contrast to spatial discretization of the state variable, we suppose that the parameter variations are more limited. For example, as a simplification we can consider piecewise-constant coefficients in the rather large regions presented in Figure 8 and defined based on physiological grounds. Clearly, more complex variation patterns can be defined for the parameters [21], but in any case we can suppose that $\theta$ is a "reasonably small" finite dimensional vector gathering all the parameters of interest in all regions. Our imperfect knowledge on the system (14) arises from the fact that we do not have any direct access to $\zeta_{x}$ and $\zeta_{\vartheta}$, since these two variables represent the specificity of the subject considered. However, we assume that we have some information on the range within which these variables may vary. In other words, if we describe the discretized counterparts $\zeta_{h}$ and $\zeta_{\theta}$ of the variations $\zeta_{x}$ and $\zeta_{\vartheta}$ as random variables over all the possible subjects, we can define covariances

$$
P_{h}=\operatorname{Cov}\left(\zeta_{h}\right), \text { and } P_{\theta}=\operatorname{Cov}\left(\zeta_{\theta}\right) .
$$

Consequently, simulating the system starting from the a priori initial conditions would result in a persistent error between the real and simulated systems all along the trajectory. We also point out that $\mathcal{A}_{h}$ may contain modeling errors (in addition to the discretization errors) that we could represent by an additional variable with an associated covariance [47].

The general principle of data assimilation then consists in using some available measurements on the system (14) to obtain a better approximation than with a direct simulation. Then, it is common in the data assimilation formalism to introduce a so-called observation operator $\mathcal{H}$ that models the measurement processes on the system. Gathering all the observations at a specific time $t$ into a variable denoted by $Z$, we have

$$
Z=\mathcal{H}(x, t)+\chi
$$

where $\chi$ represents the observation noise supposed to be an additive random process of controlled covariance $W$. Without loss of generality, we can assume that $Z$ is of finite dimension since the data are always eventually discrete. We denote by $\mathcal{H}_{h}$ the resulting discrete counterpart of the observation operator. Remark that this modeling process can be as complex as for the system itself and we will see in the next sections that the definition (16) can be too restrictive to adequately represent all the measurements processes.

From these initial definitions, data assimilation procedures fall into two major categories:

- Variational approaches in which the estimation is the result of a minimization principle based on measurement-related criteria. The most classical type corresponds to

$$
\min _{\xi_{h}, \xi_{\theta}}\left\{\mathcal{J}\left(\xi_{h}, \xi_{\theta}, t\right)=\int_{0}^{T}\left\|Z-\mathcal{H}_{h}\left(x_{h, \xi}\right)\right\|_{W^{-1}}^{2} d t+\left\|\xi_{h}\right\|_{P_{h}^{-1}}^{2}+\left\|\xi_{\theta}\right\|_{P_{\theta}^{-1}}^{2}\right\}
$$


for the class of the system defined by

$$
\left\{\begin{array}{l}
\dot{x}_{h, \xi}=\mathcal{A}_{h}\left(x_{h, \xi}, \theta_{\xi}, t\right) \\
x_{h, \xi}(0)=x_{h 0}+\xi_{h} \\
\theta_{\xi}=\theta_{0}+\xi_{\theta}
\end{array}\right.
$$

- Sequential approaches in which the observations are directly incorporated into the dynamical system in order to compensate for the initial uncertainties. The principle is then to define a gain operator $\mathcal{G}_{e}=\left(\begin{array}{c}\mathcal{G}_{x} \\ \mathcal{G}_{\theta}\end{array}\right)$ such that the modified system

$$
\left\{\begin{array}{l}
\dot{\hat{x}}_{h}=\mathcal{A}_{h}\left(\hat{x}_{h}, \hat{\theta}, t\right)+\mathcal{G}_{x}\left(Z-\mathcal{H}_{h}\left(\hat{x}_{h}, t\right)\right) \\
\hat{\hat{\theta}}=\mathcal{G}_{\theta}\left(Z-\mathcal{H}_{h}\left(\hat{x}_{h}, t\right)\right) \\
\hat{x}_{h}(0)=x_{h 0} \\
\hat{\theta}=\theta_{0}
\end{array}\right.
$$

is expected to converge over time to the target system.

In the case of linear operators $\mathcal{A}_{h}$ and $\mathcal{H}_{h}$, the most famous gain operator is given by the Kalman filter [27]. Then, the variational approach based on the criterion (17) and the sequential Kalman approach can be shown to be equivalent [6], which - indeed - proves the optimality of the Kalman filter. As regards complexity considerations, variational approaches typically require the computation of an adjoint state in order to compute the gradient of the criterion. This means that - at each minimization step - a double time integration must be performed (forward and backward in time for the direct and adjoint states, respectively) with a finite element problem solved at each time step for both states. This induces heavy computations, together with extensive storage because the direct state is needed at all time steps to solve for the adjoint state.

On the other hand, sequential approaches are very attractive because they only require the simulation of a forward dynamical system. In general, however, as described in further detail below classical sequential methods are intractable when dealing with distributed systems, because they necessitate the use - hence the computation, storage and various manipulations - of covariance matrices that have the size of the state vector and have full profile.

In this paper, we thus focus on a sequential algorithm that retains the algorithmic simplicity of filtering without requiring the computation of this covariance matrix. For the sake of clarity and compactness since we consider approximation systems, we will limit ourselves to expressions on the finite element vectors $\hat{X}$ of degrees of freedom representing the function $\hat{x}_{h}$. Therefore, the filtering estimation takes the general matrix form

$$
\left\{\begin{array}{l}
\dot{\hat{X}}=A(\hat{X}, \hat{\theta}, t)+G_{X}(Z-H(\hat{X}, t)) \\
\hat{\hat{\theta}}=G_{\theta}(Z-H(\hat{X}, t)) \\
\hat{X}(0)=X_{0} \\
\hat{\theta}=\theta_{0}
\end{array}\right.
$$

\subsubsection{Definition of a discrepancy operator}

Before presenting our data assimilation procedure, we need to define the observation operator associated with the available measurements presented in Section 2.3. We focus on kinematic observations whereas the pressure will be supposed to be a classical source term in the dynamics. We refer to Section 4.1 for details on this choice. Therefore, we assume that the only 
available observations are given by triangular meshes resulting from the segmentation of the LV endocardium and epicardium for each time frame of the Cine-MRI.

When dealing with image measurements, in general the available data cannot be modeled in the simple form (16) because it is nearly impossible to identify material points displacing from one image to another. Instead, in the case of segmented images, we can typically compare the boundary of the deformed model with the segmented surfaces and quantify the error by computing the distance fields between these computed and measured surfaces. Denoting by $\operatorname{dist}\left(y_{h}, S\right)$ the signed distance field of the boundary of the deformed simulated model to a given segmented surface $S$ and its corresponding finite element vector $\operatorname{Dist}(Y, S)$, we will thus be led to substituting this quantity for the difference $Z-H(\hat{X}, t)$ used in $(20)$. Note that we also need to deal with time sampling, meaning that we only have a discrete number of measured contours $S_{k}$ corresponding to the successive time steps $k \Delta t, 1 \leq k \leq N$, which is achieved by using an interpolation of the distance. For example, with a linear interpolation we define a so-called discrepancy operator, see [34],

$$
D(\hat{X}, t)=\left(\alpha_{k}(t) \operatorname{Dist}\left(\hat{Y}, S_{k}\right)+\left(1-\alpha_{k}(t)\right) \operatorname{Dist}\left(\hat{Y}, S_{k+1}\right)\right),
$$

with $\alpha_{k}(t)=\frac{t-t_{k+1}}{t_{k}-t_{k+1}}$ for $t \in\left[t_{k}, t_{k+1}\right]$ so that the estimator becomes

$$
\left\{\begin{array}{l}
\dot{\hat{X}}=A(\hat{X}, \hat{\theta}, t)+G_{X} D(\hat{X}, t) \\
\dot{\hat{\theta}}=G_{\theta} D(\hat{X}, t) \\
\hat{X}(0)=X_{0} \\
\hat{\theta}=\theta_{0}
\end{array}\right.
$$

Since in our case the discrepancy operator does not depend on the velocity, by a slight abuse of notation we will also write $D(\hat{Y}, t)$.

\subsubsection{State estimate}

Regarding complexity considerations, the main challenge for filtering methods is to define a tractable state gain $G_{X}$. A full Kalman filter will require for the computing of the gain $G_{X}$ to store a dense covariance matrix $P^{x x} \in \mathcal{M}_{n, n}(\mathbb{R})$ such that $G_{X}=P^{x x} H^{T} W^{-1}$. This computation is clearly intractable when $n$ grows large.

Let us concentrate on this state gain in this section and momentarily disregard the parameter estimation by supposing that there is no uncertainty in the parameter space. We will denote by $\bar{X}$ the estimator in this particular case. In [34], considering the type of observations (21), we have shown that we can define an adequate $G_{X}$ that does not necessitate the computation of dense covariance matrices. Instead, we use the specificity of the mechanical system equations to formulate an effective filter that converges to the reference system, as was mathematically substantiated for small errors with the analysis of the linearized system in [34]. The estimator for perfect parameters then consists in incorporating a filtering term in the equation relating displacements and velocities. This filtered equation takes the following form in continuous time [34]

$$
\dot{\bar{Y}}=\bar{V}-\gamma\left(\epsilon K_{e l}+\frac{\partial D^{T}}{\partial Y} M_{\Gamma_{m}} \frac{\partial D}{\partial Y}\right)^{-1} \frac{\partial D^{T}}{\partial Y} M_{\Gamma_{m}} D(\bar{Y}, t)
$$

where $\gamma$ is a gain parameter, $K_{e l}$ denotes a linearized stiffness matrix - e.g. around the reference configuration or the current trajectory $-\epsilon$ a "small" regularization parameter, and $M_{\Gamma_{m}}$ the 
matrix associated with surface (scalar) dot product on the observed boundary $\Gamma_{m}$ and normalized so that for the specific displacement vector $Y_{1}$ associated with a constant displacement field having the 3 components equal to unity:

$$
Y_{1}^{T} M_{\Gamma_{m}} Y_{1}=1 .
$$

The time discretization of this filtered equation is provided in Appendix A, in the same spirit as for the direct model, see Eq. (31), and this is the time-discrete system that will be considered in the next section for the additional stage of parameter identification.

\subsubsection{Joint state-parameter estimate}

In order to apply the joint state-estimation procedure presented in [32], we summarize the discretized version of the state estimator defined in (31) of the Appendix as

$$
\left\{\begin{array}{l}
\bar{X}^{n+1}=A_{n+1 \mid n}^{G}(\theta)\left(\bar{X}^{n}\right)+R^{n} \\
\bar{X}^{0}=X_{0} \\
\theta=\theta_{0}+\zeta_{\theta}
\end{array}\right.
$$

where for all $n, \bar{X}^{n}=\left(\bar{Y}^{n} ; \bar{V}^{n} ;\left\{\bar{e}_{c, h}^{n}\right\} ;\left\{\bar{\tau}_{c, h}^{n}\right\} ;\left\{\bar{k}_{c, h}^{n}\right\}\right)$. This space- and time-discrete model is not computable only because $\zeta_{\theta}$ is unknown. Following exactly [32] we then apply a so-called unscented Kalman filter (UKF) introduced in [26], albeit here reduced to the parametric space for a specific choice of the so-called sigma-points as inspired from [39, 20], see also [51] for similar ideas applied to the estimation of electrophysiology parameters.

Given adequate sampling rules, we precompute the corresponding symplectic unitary sigmapoints $I_{[i]}, 1 \leq i \leq p+1$. We associate the corresponding weights collected in a diagonal matrix $D_{\alpha}$ and perform

\section{- Sampling:}

$$
\left\{\begin{array}{l}
C_{n}=\sqrt{\left(U^{n}\right)^{-1}} \\
\hat{X}_{[i]}^{n^{+}}=\hat{X}^{n^{+}}+L_{X}^{n}\left(C^{n}\right)^{T} I_{[i]}, \quad 1 \leq i \leq p+1 \\
\hat{\theta}_{[i]}^{n^{+}}=\hat{\theta}^{n^{+}}+L_{\theta}^{n}\left(C^{n}\right)^{T} I_{[i]}, \quad 1 \leq i \leq p+1
\end{array}\right.
$$

\section{- Prediction:}

$$
\left\{\begin{array}{l}
\hat{X}_{[i]}^{n+1^{-}}=A_{n+1 \mid n}^{G}\left(\hat{X}_{[i]}^{n^{+}}, \hat{\theta}_{[i]}^{n+}, t^{n+1}\right) \\
\hat{X}^{n+1^{-}}=E_{\alpha}\left(\hat{X}_{*}^{n+1^{-}}\right) \\
\hat{\theta}^{n+1^{-}}=\hat{\theta}^{n+1^{+}}
\end{array}\right.
$$

\section{- Correction:}

$$
\left\{\begin{array}{l}
L_{X}^{n+1}=\left[\hat{X}_{*}^{n+1^{-}}\right] D_{\alpha}\left[I_{*}\right]^{T} \in \mathcal{M}_{N, p} \\
L_{\theta}^{n+1}=\left[\theta_{*}^{n+1^{-}}\right] D_{\alpha}\left[I_{*}\right]^{T} \in \mathcal{M}_{p} \\
\Gamma_{[i]}^{n+1}=D\left(X_{[i]}^{n+1^{-}}, t^{n+1}\right) \\
L_{\Gamma}^{n+1}=\left[\Gamma_{*}^{n+1}\right] D_{\alpha}\left[I_{*}\right]^{T} \\
U^{n+1}=\mathbb{1}+\gamma_{\chi}\left(L_{\Gamma}^{n+1}\right)^{T}\left(W_{n+1}\right)^{-1} L_{\Gamma}^{n+1} \in \mathcal{M}_{p} \\
\hat{X}^{n+1^{+}}=\hat{X}^{n+1^{-}}-\gamma_{\chi} L_{X}^{n+1}\left(U^{n+1}\right)^{-1}\left(L_{\Gamma}^{n+1}\right)^{T}\left(W_{n+1}\right)^{-1} E_{\alpha}\left(\Gamma_{*}^{n+1}\right) \\
\hat{\theta}^{n+1^{+}}=\hat{\theta}^{n+1^{-}}-\gamma_{\chi} L_{\theta}^{n+1}\left(U^{n+1}\right)^{-1}\left(L_{\Gamma}^{n+1}\right)^{T}\left(W_{n+1}\right)^{-1} E_{\alpha}\left(\Gamma_{*}^{n+1}\right)
\end{array}\right.
$$




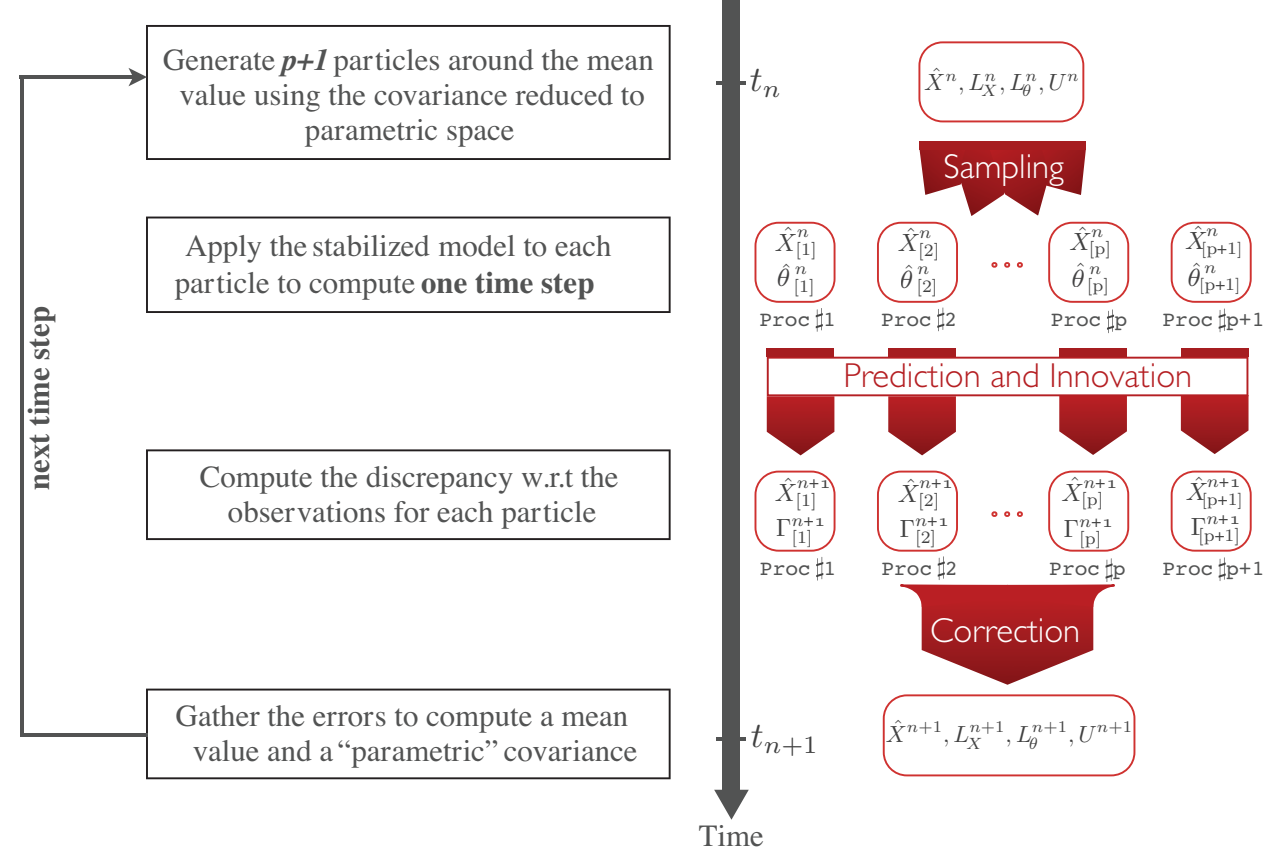

Figure 5: Parallel algorithm for joint state-parameter estimation

where for every set of particles $X_{[i]}, 1 \leq i \leq p+1,\left[X_{*}\right] \in \mathcal{M}_{N, p+1}$ denotes the concatenation of the $(p+1)$ column vectors arranged side by side, and $E_{\alpha}\left(X_{*}\right)=\sum_{1 \leq i \leq p+1} \alpha_{i} X_{[i]}$, while $W_{n+1}$ denotes the discrete-time noise covariance.

This algorithm is fully parallel and can be interpreted in the following manner, as schematized in Figure 5. From given mean value and covariance estimates of the state and parameter at time $t_{n}$, we create $p+1$ particles centered around the estimate mean value at a distance given by the standard deviation extracted from the covariance. These particles are sent to various processors where the model $(31)$ - which includes the state estimate - performs a forward computation of one time step. Furthermore, for each simulated particle we compute the discrepancy induced by the distance from the generated contours and the given segmentations. The resulting state variables and discrepancy are sent back to the master processor. There, a new prediction mean value and covariance are computed and then corrections are introduced in these predictions from the discrepancy computations. With this parallel approach, the estimation computational cost is comparable to that of a simple forward simulation of the stabilized model, the complexity of which was discussed in [34]. By contrast, a variational estimation would require a significant number of successive iterations (typically over a hundred) of both forward and adjoint simulations.

We emphasize that - by construction of the filtering procedure - the estimated parameter values evolve during the simulation period - corresponding to a complete heartbeat in our case - and of course the actual estimation is achieved with the final values. Hence, we expect these estimation curves to actually stabilize in the course of the simulation, since non-converging parameter estimation would be likely to indicate persistent modeling error, indeed. 

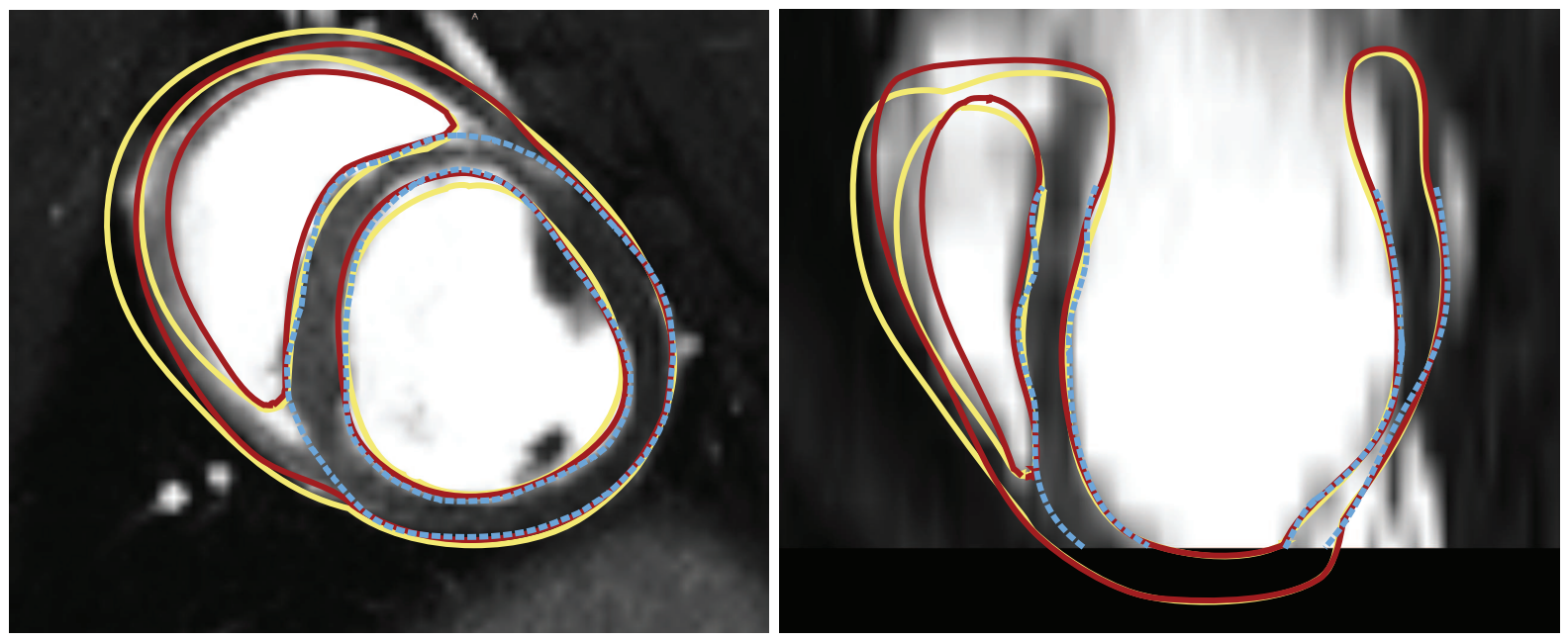

Figure 6: Comparison of the original initial mesh (yellow) and the mesh obtained by running the state estimation with a large gain $\gamma$ (red) with the segmentation (dashed blue)

\section{Regional contractility estimation}

In this section, we will describe and assess the use of the above-presented modeling and data assimilation components to estimate localized contractility parameters, with constant values over pre-defined subregions. Hence, in our case in the data assimilation procedure the dimension $p$ of the parameter space corresponds to the number of regions employed. This estimation objective is selected in adequacy with the type of pathology considered, namely, major infarction without any specific secondary pathology - in particular as regards electrical activation timings. In such a case we can expect contractility variations in our model to correctly represent the post-infarct behavior [11].

\subsection{Modeling setup}

Before presenting the detailed estimation objectives and setup, we should mention that an important prerequisite is that the biomechanical heart model be adequately pre-calibrated in order to present a physiological behavior globally compatible with the data. The calibration procedure for this specific model is summarized in [11] and consists in adjusting one by one the key biophysical parameters of the model at the baseline stage - with constant values over the whole myocardium - by making use of the available data, namely, catheter pressures and MR images, at specific phases of the heart cycle. In particular, passive stiffness is calibrated based on ventricular filling induced by atrial contraction, while contractility is globally adjusted in systole in order to obtain a correct ejection fraction. In addition, the fiber angles can be adapted at this stage to produce a motion pattern in better accordance with image data, in particular as regards the respective amounts of longitudinal versus radial shortening.

The following considerations are also of importance in the model preparation:

- The initial geometry needs to be as close as possible to the observation, in order to reduce the initial error. This is why the computational mesh used for the joint stateparameter estimation was created from the original segmented mesh, and corrected by running the state estimator with a very large gain $\gamma$ during one complete cardiac cycle. Hence, the estimation procedure was used here for image processing purposes only in a 

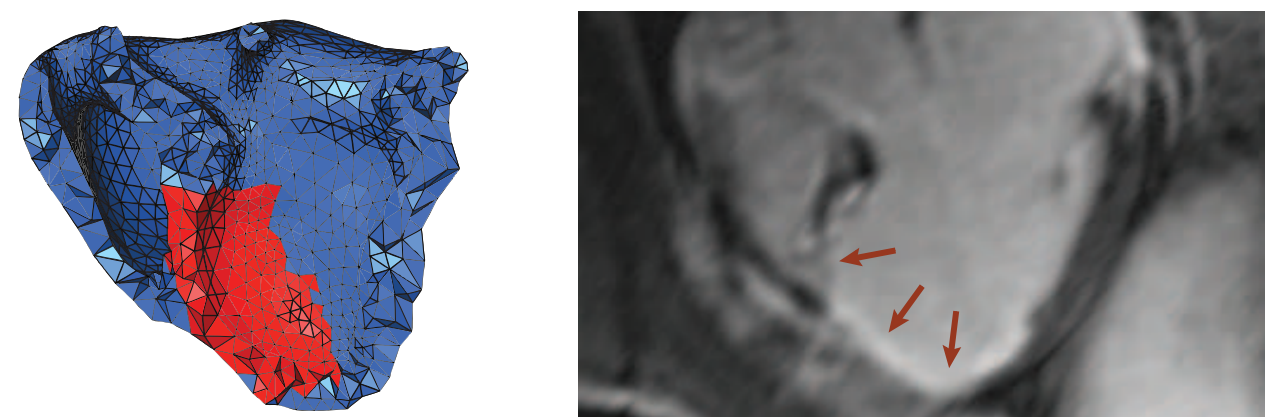

Figure 7: Infarct at $T_{0}+38$ : in the mesh (left, in red) as segmented from the late enhancement MR images (right, red arrows)

first stage - namely, without any particular concern for physical predictivity. Figure 6 shows a comparison of the original initial mesh, the corrected mesh by means of the state estimation with the large gain $\gamma$ and the observation surfaces. This corrected mesh was then used for the joint state-parameter estimations.

- The initiation of the model contraction and of its relaxation needs to be as synchronous as possible with the observations. Any offset in the electrical activation of the model can cause sudden changes of the estimated parameters in arbitrary directions. The electrical activation pattern is often impaired by the myocardial infarction, and any large dyssynchrony in the contraction should be taken into account in the model activation. In our case, however, the ECG obtained for each stage of the study showed no irregularity and the contractions were visibly synchronous. For this reason, we were able to keep using the same - physiological - electrical activation pattern in all stages, and then the variations of estimated contractility parameters summarize - via the product $\sigma_{0}|u|_{+}$in $(9)$ - the combined impact of the pathology on the activation signal intensity and on the ability of the tissue to respond to this signal.

- In our estimation trials we use prescribed pressures, based on the clinical measurements, namely, without the Windkessel models. However, rather than directly using the pressure measurements which are noisy and imperfectly synchronized with the electrical activation, we exported the pressure values from the pre-calibrated direct model including the Windkessel components. This provided pressure profiles in very good agreement with the measurements, albeit smoother and perfectly registered with the electrical activation.

\subsection{Estimation setup}

Our aim is to estimate some contractility parameter values regionalized according to two different geometric subdivision schemes. The first subdivision applied to the stages $T_{0}+10$ and $T_{0}+38$ predefines two regions based on a segmentation of the late enhancement imaging projected in the model, see Figure 7. This two-region subdivision into infarcted and non-infarcted parts will be primarily used in the calibration of the estimation procedure. In addition, at each stage we defined a subdivision of the left ventricle myocardium into 6 circumferential parts, corresponding in their upper third to the basal segments of the standard 17-segment AHA 

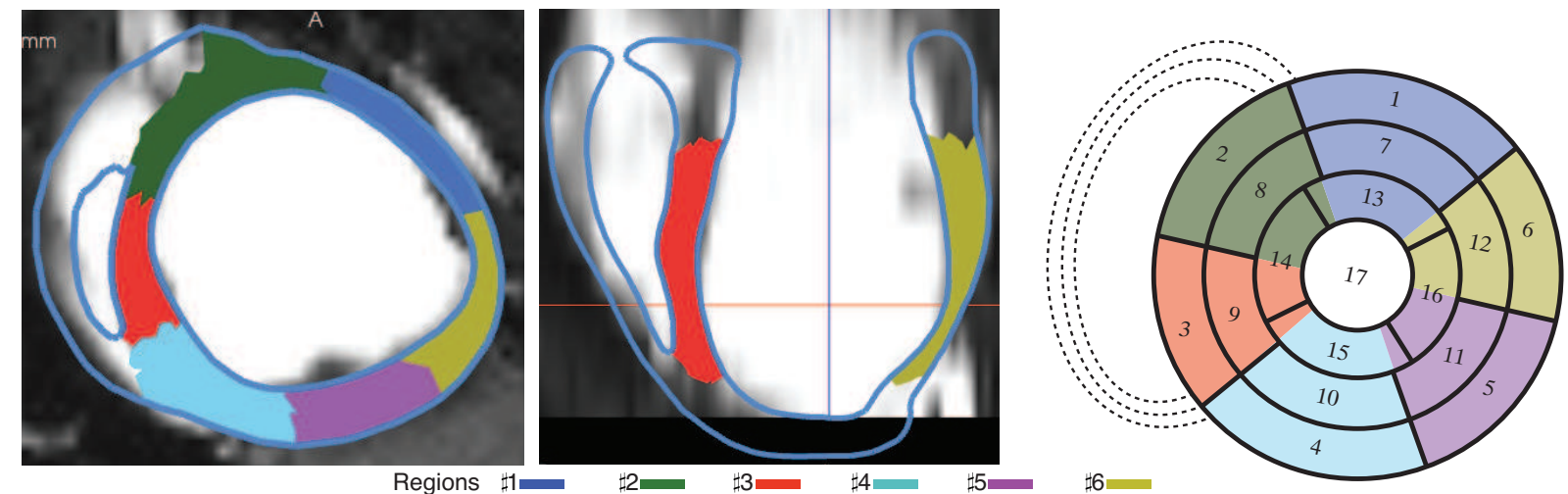

Figure 8: Mesh of $T_{0}+38$ subdivided into $6+1$ volumic regions and plotted within MRI (left and center) - myocardium not in color representing the non-segmented regions with volume reference $\sharp 0$ - and correspondence with AHA segments in bull's eye schematic (right)

\begin{tabular}{|c||c|l|c|c|c|}
\hline Region & AHA segments & Name & Region vol. & Infarct \% & Thickening \\
\hline \hline 0 & - & non-segmented & $121 \mathrm{~mL}$ & $3 \%$ & - \\
1 & $1+7^{*}$ & anterior & $11 \mathrm{~mL}$ & $45 \%$ & $15 \%$ \\
2 & $2+8^{*}$ & antero-septal & $15 \mathrm{~mL}$ & $64 \%$ & $0 \%$ \\
3 & $3+9^{*}$ & infero-septal & $10 \mathrm{~mL}$ & $20 \%$ & $7 \%$ \\
4 & $4+10 *$ & inferior & $16 \mathrm{~mL}$ & $0 \%$ & $52 \%$ \\
5 & $5+11 *$ & infero-lateral & $12 \mathrm{~mL}$ & $0 \%$ & $63 \%$ \\
6 & $6+12 *$ & antero-lateral & $13 \mathrm{~mL}$ & $0 \%$ & $49 \%$ \\
\hline
\end{tabular}

Table 1: Description of $6+1$ region mesh subdivision in relation to AHA segments, corresponding volumes, proportion of infarcted tissue, and measured thickening in each region at $T_{0}+38$

\begin{tabular}{|c||c|c|c|c|c|c|c|c|}
\hline AHA segments & 1 & 2 & 3 & 4 & 5 & 6 & 7 & 8 \\
\hline Volume $(\mathrm{mL})$ & 5.2 & 5.6 & 4.3 & 6.7 & 6.0 & 6.3 & 3.6 & 4.7 \\
\hline Infarct \% & 41 & 45 & 3 & 0 & 0 & 0 & 35 & 72 \\
\hline \hline AHA segments & 9 & 10 & 11 & 12 & 13 & 14 & 15 & 16 \\
\hline Volume (mL) & 2.8 & 5.3 & 4.1 & 4.3 & 3.6 & 6.1 & 5.2 & 3.6 \\
\hline Infarct \% & 13 & 0 & 0 & 0 & 84 & 59 & 0 & 6 \\
\hline
\end{tabular}

Table 2: Volumes and infarct proportions for each AHA segment at $T_{0}+38$ 

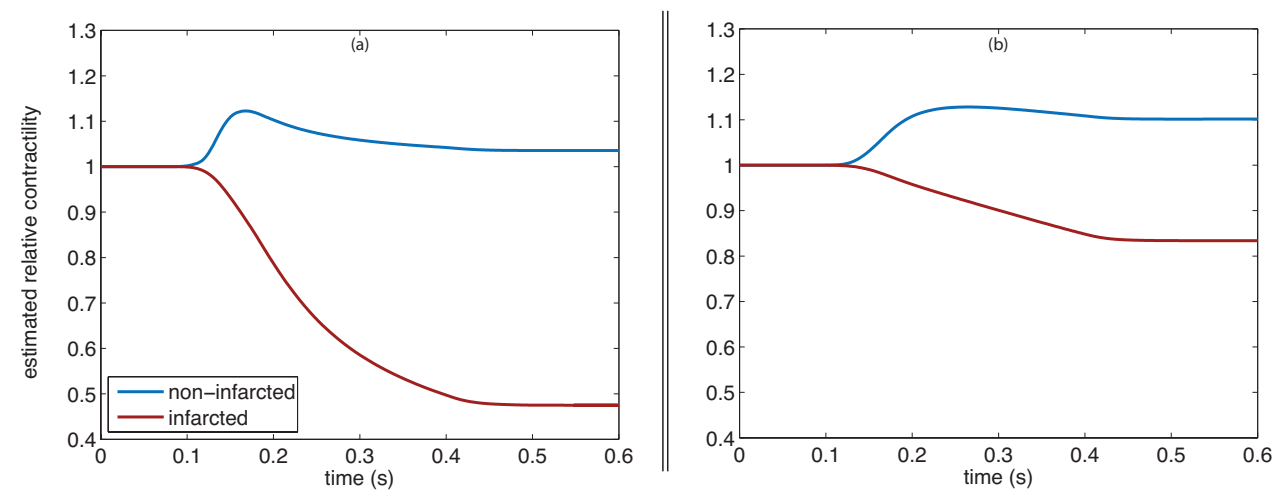

Figure 9: Two-region estimation at stage $T_{0}+38$ using synthetic observation with two levels of measurement noise covariance $\left(\right.$ left $w_{o b s} \sim(1.5 \mathrm{~mm})^{2}$ and right $\left.w_{o b s} \sim(4.8 \mathrm{~mm})^{2}\right)$

subdivision [10], see Figure 8 and also the description in Table 1. This $6+1$ region subdivision - counting as region $\sharp 0$ the apex, the base and the right ventricle that were not segmented, see Figure 8 - will be prioritarily used in the estimation trial with clinical data in order to assess the capability of the procedure to both localize and quantify the infarct-related parameters. We further give in Table 1 the proportion of tissue marked by the late enhancement in each region, together with systolic thickening indices obtained by considering three short axis slices, manually measuring thickening at three locations in each AHA segment, and weighing the results by the AHA segment distribution within each region. Finally, we will provide some estimation results associated with the complete AHA subdivision, hence we likewise list the corresponding infarcted proportions in Table 2. In order to avoid any confusion with the $6+1$ region subdivision, we will systematically refer to "segment $\sharp n$ " versus "region $\sharp p$ " in the sequel.

Before being able to apply the above data assimilation procedure to estimate regional contractility values of the model based on the Cine-MRI, we need to calibrate the key parameters used in this procedure, namely, in particular the measurement noise covariance and the state estimator gain $\gamma$. To that purpose we will employ so-called synthetic measurements, i.e. in our case contour meshes directly generated from a given simulation of the model, so as to fully control the ground truth in the estimation. This will be done using the anatomical model corresponding to the infarcted stage $T_{0}+38$.

In order to calibrate the measurement noise covariance, we generated synthetic measurements by reducing the model contractility in the infarcted region - as segmented from the late enhancement MRI, see Figure 7 - by a factor four compared to a reference homogeneous value obtained by calibrating the whole model in the baseline (healthy) stage. We then started the state-parameter estimation procedure with two contractility values to be estimated, associated with the infarcted region and the rest of the tissue, namely, according to the 2-region subdivision. Of course, the contractility parameters were initialized at the reference value at the start of the estimation, and we compared the estimation results obtained with several choices of measurement noise in the form:

$$
\left(W_{n}\right)^{-1}=w^{-1} M_{\Gamma_{m}}, \quad \forall n,
$$

with $w$ a parameter to be defined representing the square of the standard deviation of the distance error. Considering that the computational time step $\Delta t^{\text {sim }}$ used in the estimation is very different from - indeed, much smaller than - the imaging time sampling $\Delta t^{\text {obs }}$, we need to rescale this parameter. Assuming that the underlying measurement error process has time-wise 


\begin{tabular}{|c||c|c|c|c|}
\hline Region & 1st run & 2nd run & 3rd run & Ref. \\
\hline \hline Healthy & 1.04 & 1.08 & 1.09 & 1 \\
\hline Infarct & 0.48 & 0.33 & 0.29 & 0.25 \\
\hline
\end{tabular}

Table 3: Two-region estimation at stage $T_{0}+38$ using synthetic observation with repeated runs

white noise characteristics, this rescaling should be performed according to the standard rule

$$
\Delta t^{s i m} w=\Delta t^{o b s} w_{o b s} .
$$

In our case, with $\Delta t^{o b s} \sim 26 \mathrm{~ms}$ and $\Delta t^{\text {sim }} \sim 1 \mathrm{~ms}$, we tested the following values of $w$ :

- $w=6.110^{-5} \mathrm{~m}^{2}$, corresponding to $w_{\text {obs }} \sim(1.5 \mathrm{~mm})^{2}$ according to Eq. (26);

- $w=6.110^{-4} \mathrm{~m}^{2}$, corresponding to $w_{\text {obs }} \sim(4.8 \mathrm{~mm})^{2}$.

In both cases we took $P_{\theta 0}=\frac{1}{2} \mathrm{Id}$, thus defined to have the mean of the Euclidean norm of the parameter uncertainty vector scaled to unity. The estimation results are shown in Figure 9. Note that - by construction of filtering estimation - the estimated values vary during the simulation window corresponding here to the heartbeat, and that they stabilize before the end of the simulation, indeed. Moreover, as expected, when increasing $w$ more weight is placed in the estimation on the a priori parameter values, hence the parameter estimation sensitivity is decreased. Considering both the reference values used for the parameters - namely, 0.25 in the infarcted area and 1 elsewhere - and the expected accuracy of the measurement process, the value $w=6.110^{-5} \mathrm{~mm}^{2}$ appears to be a good compromise, and will be used in the sequel. We remark that, with this calibration, we still do not obtain good relative accuracy for the contractility in the infarcted region, as the final estimated value is 0.48 (compared to the 0.25 reference). However, this is consistent with the fact that data assimilation intrinsically balances the expected uncertainties in the data with those assumed in the a priori values prescribed for the estimated parameters. Here, the fact that the estimated non-infarcted contractility is much more accurate (1.04 for 1 ) can be explained by the starting value being much closer to the ground truth. If we want to change this uncertainty balance assumption, we can place more confidence in the data by varying the covariance scalings. Alternatively, we can also use data over a longer time window when available - as is frequently the case in our situation, since several heartbeats are generally acquired. With synthetic measurements this can be simulated by restarting the estimation with the parameter values obtained in the previous run. Table 3 displays the results obtained in our case after several runs, with an improved overall accuracy as the estimation window is increased.

With a view to calibrating the gain of the state estimator in an experiment closest to the regional estimation objective, we next considered the $6+1$ region subdivision. We then generated synthetic measurements by prescribing in each region a contractility value consistent with the extent of the infarct, and tested the estimation procedure using these measurements with several values of the state filter gain. We show the results in Figure 10, where $\gamma_{0}$ denotes a reference gain value computed by a pole analysis on the model linearized around the reference configuration [34]. As expected, when increasing the gain we reduce the sensitivity of the parameter estimation, because the discrepancy quantity on which the parameter filter is applied is then reduced. Nevertheless, it is also important to use a sufficiently large value of the gain in order to maintain the model reasonably close to the data, for the parameter sensitivities to be accurately approximated in the procedure. Therefore, in the sequel we will use the intermediate value $\gamma=20 \gamma_{0}$. As a final verification, we show in Fig. 11 the estimation results obtained 

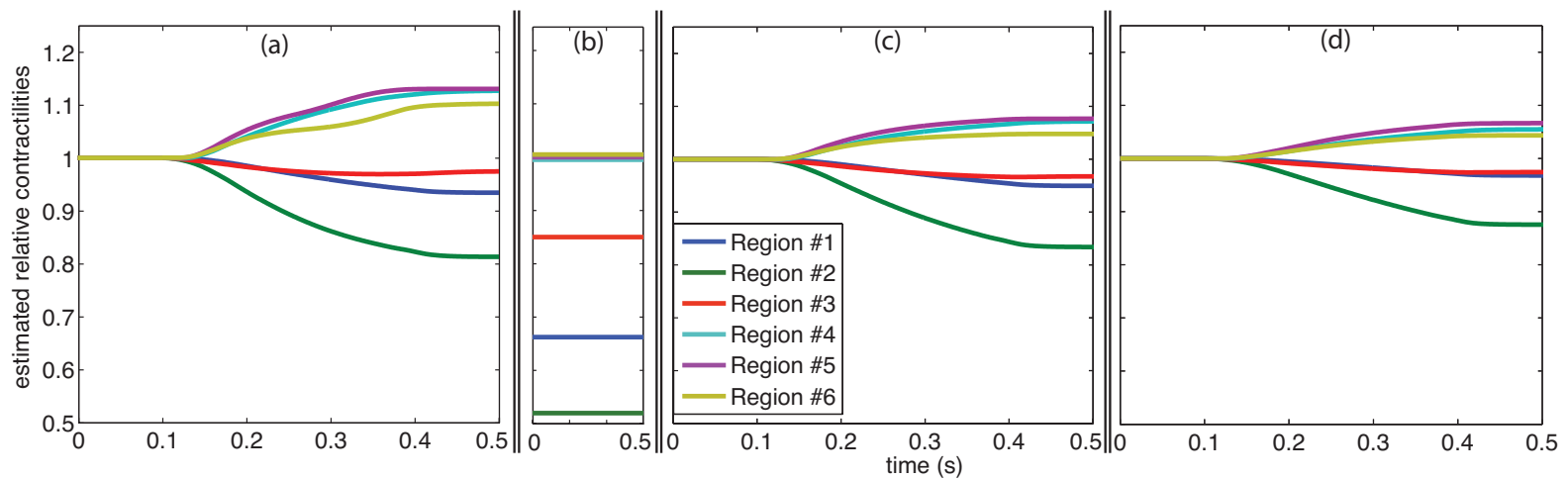

Figure 10: Regional contractility estimation at stage $T_{0}+38$ using synthetic observation and varying the state filter gain $\gamma$. (a) $\gamma=8 \gamma_{0}$ (b) ground truth reference (c) $\gamma=20 \gamma_{0}$ (d) $\gamma=40 \gamma_{0}$
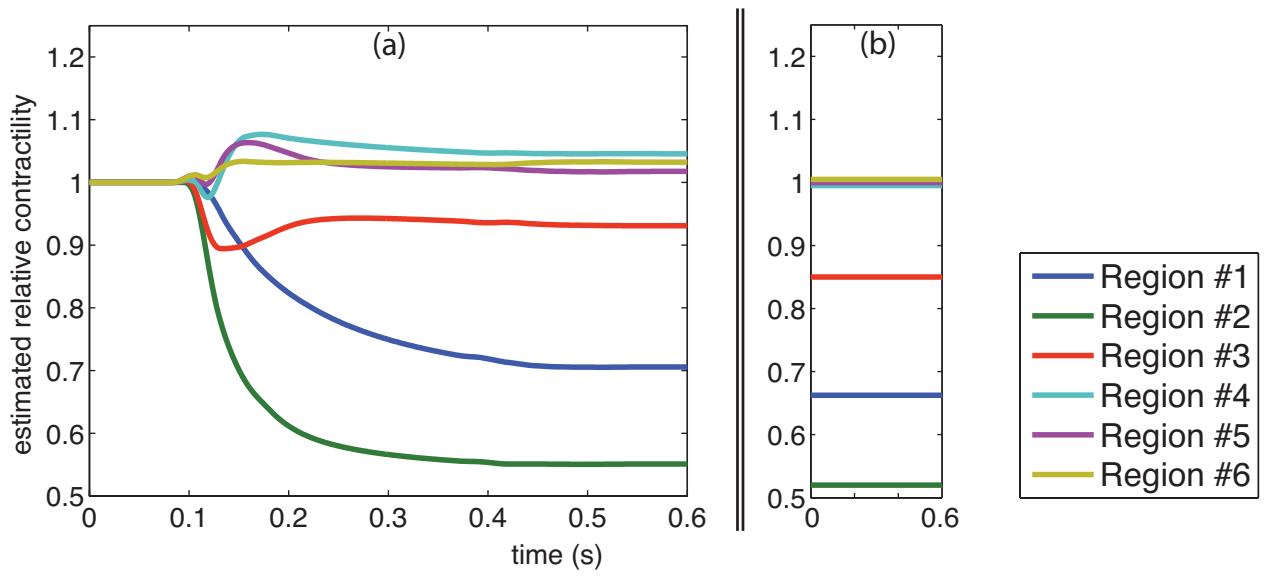

Figure 11: (a) Regional contractility estimation at stage $T_{0}+38$ using synthetic observation with a very low level of measurement noise $\left(w_{o b s} \sim(0.15 \mathrm{~mm})^{2}\right)$ (b) Reference values

with the same synthetic measurements and still $\gamma=20 \gamma_{0}$ when using a very low measurement covariance $\left(w=6.110^{-7} \mathrm{~m}^{2}\right.$, namely, $\left.w_{o b s} \sim(0.15 \mathrm{~mm})^{2}\right)$. In this case, we see that the final estimation result is accurate, hence also that the parameters of interest are identifiable in this setup, indeed, see also the discussion section on this issue of observability.

\subsection{Results}

Figures 12(a,b,c) display the estimation results obtained with the real data - namely, the actual segmented Cine-MR image sequence - at each of the three stages. For completeness, we summarize the main numerical parameter values used in Table 4. In the baseline case, the contractility values do not vary more than by $10 \%$ in the course of the estimation. We can see that the contractility values are quite comparable between the baseline stage and $T_{0}+10$, except for regions $\sharp 2$ and $\sharp 3$ in which we observe a drop of contractility of the order of $10 \%$, and for region $\sharp 1$ where the contractility rises by about $10 \%$. At stage $T_{0}+38$, a more pronounced drop of contractility in the regions $\sharp 2$ and $\sharp 3$ is obtained, together with an increase in regions $\sharp 5$ and $\sharp 6$ - which may be caused by a compensation hypertrophy. These estimation results are globally consistent with the late enhancement data - recall Table 1 - except for what concerns 

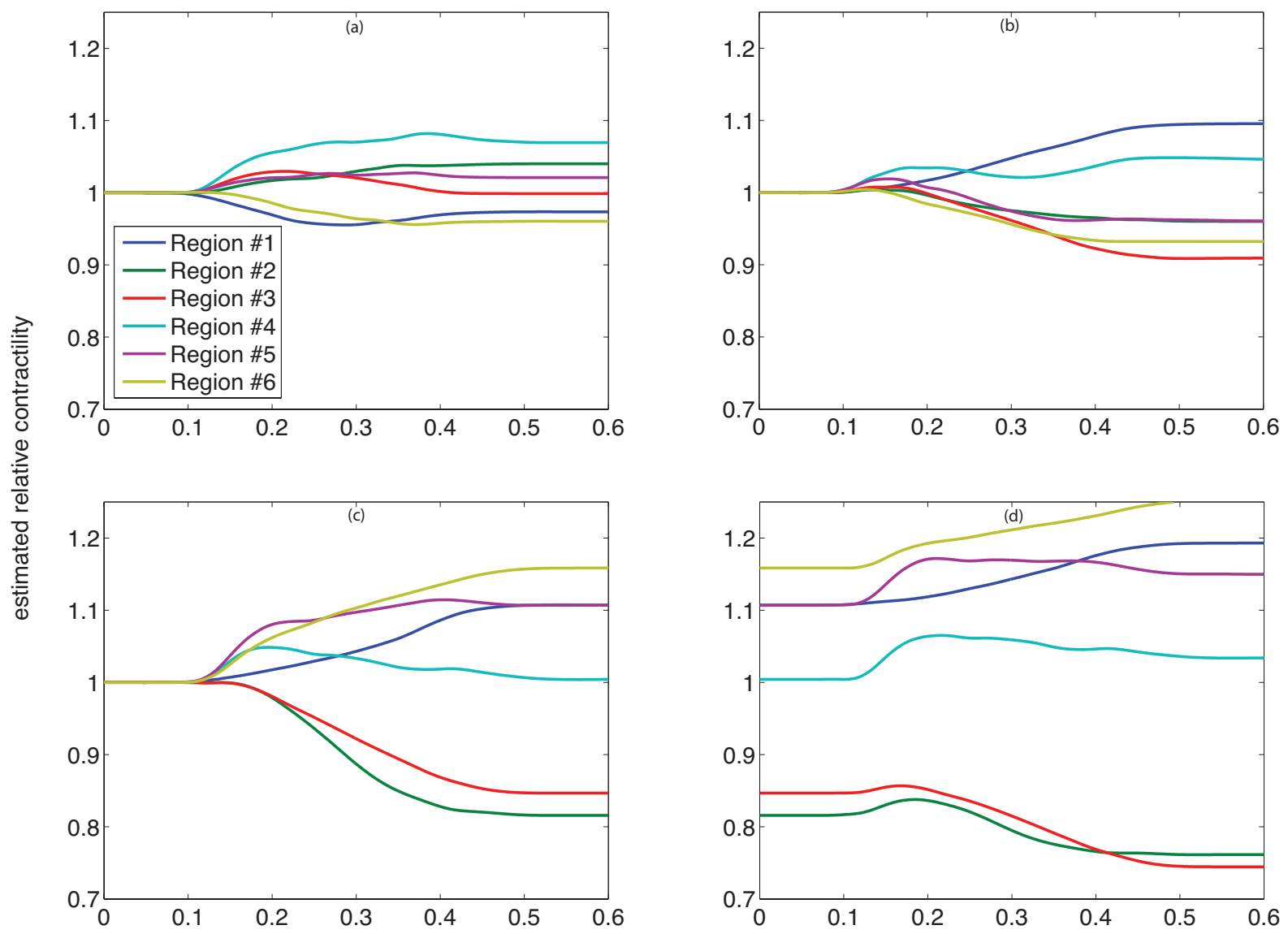

time (s)

Figure 12: Regional contractility estimation at each stage: (a) Baseline (b) $T_{0}+10$ (c) $T_{0}+38$ (d) Second run of estimation for stage $T_{0}+38$

\begin{tabular}{|c|c||l|}
\hline Parameter & Value & Note \\
\hline \hline$\gamma$ & $20 \gamma_{0}$ & state estimator gain \\
$w\left(\mathrm{~m}^{2}\right)$ & $6.110^{-5}$ & level of expected noise in the observations \\
$P_{\theta 0}$ & $\frac{1}{7} \mathrm{Id}$ & diagonal covariance matrix \\
\hline$\kappa_{1}(\mathrm{~Pa})$ & $5 \cdot 10^{3}$ & passive stiffness (first modulus) \\
$\kappa_{2}(\mathrm{~Pa})$ & 40 & passive stiffness (second modulus) \\
$\kappa(\mathrm{Pa})$ & $5 \cdot 10^{5}$ & passive stiffness (bulk) \\
\hline
\end{tabular}

Table 4: Main parameter values used in the simulation and estimation procedures 

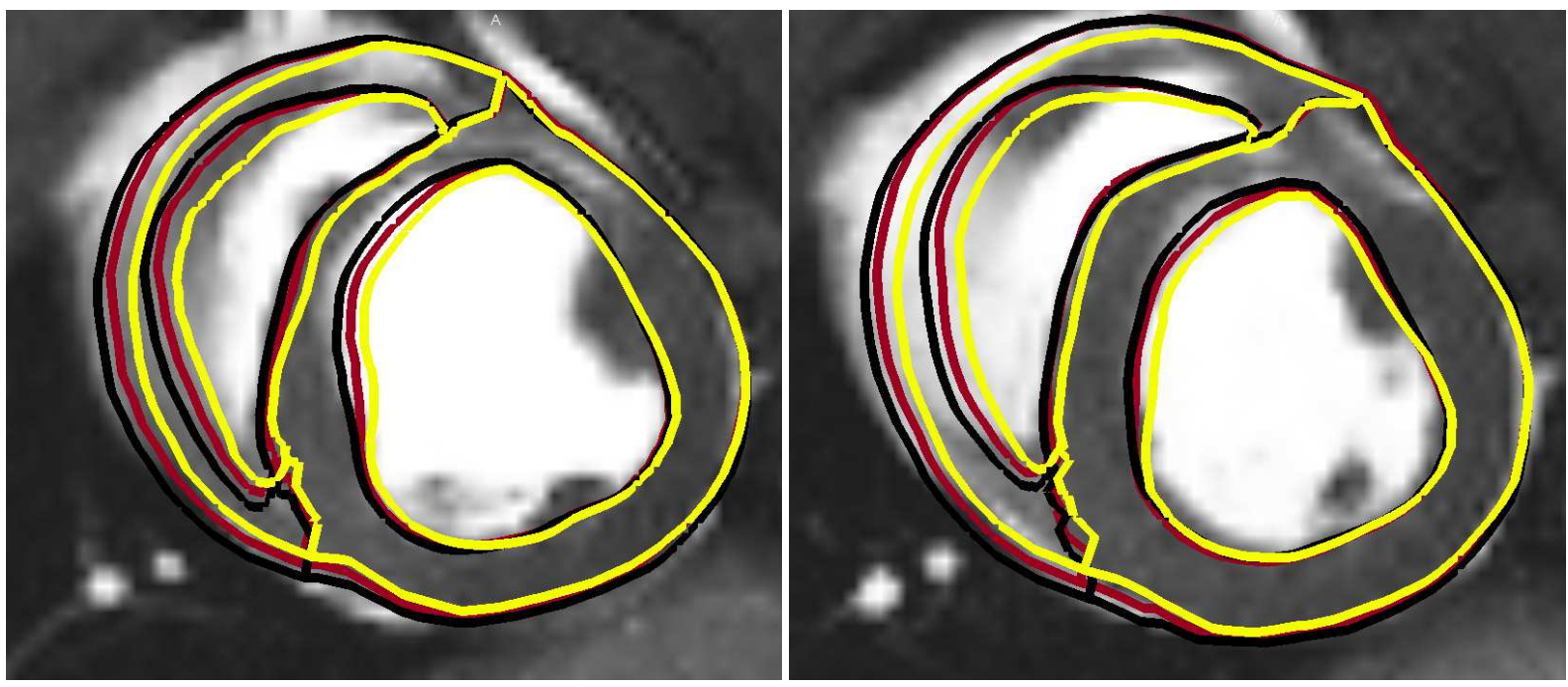

Figure 13: Two slices of the end-systolic phase at stage $T_{0}+38$. Comparison of direct model with constant contractility (yellow contour), direct model using the estimated values of contractility (red), joint state-parameter estimation (black)
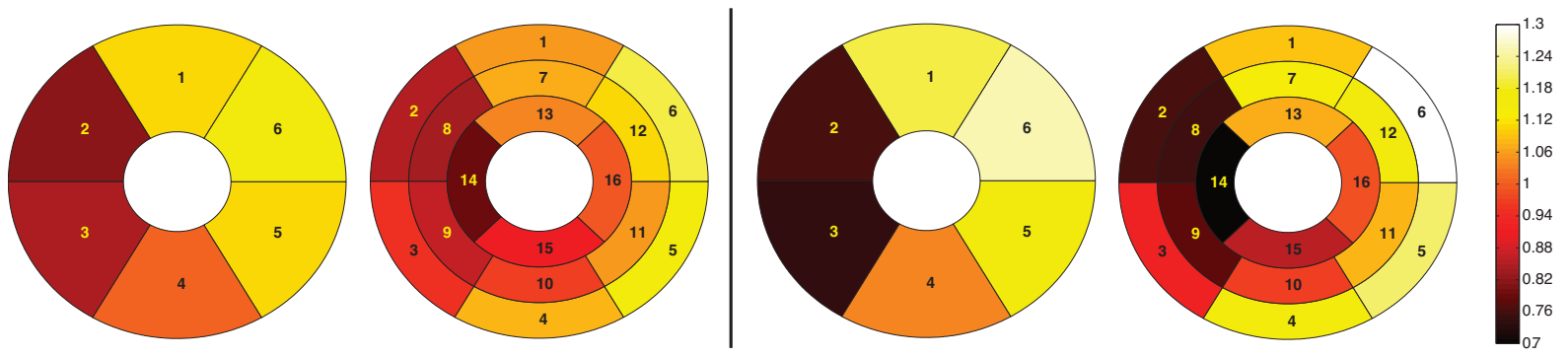

Figure 14: Estimated contractilities at $T_{0}+38$ in 6 regions versus 16 AHA segments after first (left) and second (right) run

region $\sharp 1$, which will be further discussed in Section 5 .

In Figure 12(d) we also show the estimation results obtained for stage $T_{0}+38$ when restarting the estimation procedure with the contractility values obtained at the end of the first pass visualized in Figure 12(c), and with the same measurements. This demonstrates that the parameter estimation is already quite converged - and stable - when completing the first pass.

Next, as a direct validation of the estimated contractility parameters, we performed direct simulations for stage $T_{0}+38$ using these estimated parameter values. In Figure 13 we compare the model contours corresponding to direct simulations with the constant contractility, the direct model with the estimated contractility and the joint state-parameter estimation. As expected, the best match with the data was obtained with the joint state-parameter estimation. Nevertheless, although the direct model with the estimated contractility parameters does not exactly coincide with the data, the estimated parameters clearly correct the direct model in a most adequate manner. Note in particular that the right ventricle behavior is much improved, whereas this part of the myocardium is not included in the discrepancy operator - hence it is not used in the filtering corrections - which is a sign that model predictivity is enhanced.

Finally, we have performed some estimation computations based on the complete AHA subdivision, namely, with one contractility value for each AHA segment. We observed a similar 
convergence in the estimation sequential process, but for the sake of visual clarity we only display in Figure 14 - in a bull's eye format - the final estimated values obtained. In this figure we also compare these estimation results with the previous 6-region estimations to assess their consistency, see the discussion in the next section.

\section{Discussion}

Regarding the intrinsic capabilities and limitations of the estimation procedure, some valuable insight can be gained by assessing the observability by means of the observability Gramian matrix $\Upsilon$ of the parameters [53], given here by [33]

$$
\Upsilon=\sum_{n}\left(L_{\theta}^{n}\right)^{-T}\left(L_{\Gamma}^{n}\right)^{T}\left(W_{n}\right)^{-1} L_{\Gamma}^{n}\left(L_{\theta}^{n}\right)^{-1}
$$

The error in the estimated parameter $\tilde{\theta}=\hat{\theta}-\theta_{\text {ref }}-$ with $\theta=\left(\theta_{0}, \ldots, \theta_{6}\right)^{T}$ the components of the parameter $\theta$ associated with the various regions - then satisfies

$$
\tilde{\theta}=\Upsilon^{-1} \varepsilon,
$$

with $\varepsilon=\left(\varepsilon_{0}, \ldots, \varepsilon_{6}\right)^{T}$ arising from various error source terms including measurement and modeling errors. Hence, both low observability in $\Upsilon^{-1}$ and large input errors in $\varepsilon$ lead to increased estimation errors. We thus obtained observability indices for each region by computing mean absolute values of each component of $\tilde{\theta}$ inferred from the rule (27) considered over large samples of random noise $\varepsilon$ with each component taken independent and normalized in $[-0.5,0.5]$, namely,

$$
E\left(\left|\tilde{\theta}_{i}\right|\right) \approx \frac{1}{N_{s}} \sum_{j=1}^{N_{s}}\left|\tilde{\theta}_{i}^{(j)}\right| .
$$

Table 5 shows these observability indices obtained both for the estimations on synthetic data corresponding to Fig. 10(c) and on real data corresponding to Fig. 12(c). As we can see in the table, the region $\sharp 1$ (the LV anterior wall) corresponds to the less observable part. A most likely interpretation is that this is due to the artificial boundary condition applied on the adjacent epicardial surface and which induces an added stiffness effect by which the sensitivity with respect to contractility variations decreases. The low observability of region $\sharp 3$ (inferior part of the septum) compared to region $\sharp 2$ may be interpreted by the fact that the latter is directly adjacent to the boundary conditions, hence a change of contractility in region $\sharp 2$ has a large impact on the whole septum motion - including region $\sharp 3$ - whereas the contractility in region $\sharp 3$ is bound to have a more limited impact.

Concerning our comparisons between estimated contractility values and proportions of infarcted tissue, a general word of caution is in order since - in particular - the latter indices were computed based on manual segmentation and registration of late enhancement images, hence do not give an "absolute reference". Nevertheless, in the case of region $\sharp 1$ the estimated contractility values are clearly in excess, as confirmed by the thickening indices given in Table 1. For this specific region, in addition to the high error index provided by the observability Gramian - recall Table 5 - the already mentioned added stiffness effect produced by the adjacent artificial boundary conditions is likely to also have an impact as a modeling error. Namely, this stiffening tends to be compensated by the estimation, inducing a non-physiological increase of the estimated contractility. By implementing a new type of boundary condition enabling the sliding of some parts of the epicardium along the parietal pericardium, we would expect to obtain a more physiological thickening of the anterior wall in the direct modeling. However, such sliding 


\begin{tabular}{|c||c|c|}
\hline Region & synth. obs. & real obs. \\
\hline \hline 0 & 0.25 & 0.23 \\
\hline 1 & 0.71 & 0.75 \\
\hline 2 & 0.32 & 0.31 \\
\hline 3 & 0.59 & 0.57 \\
\hline 4 & 0.18 & 0.18 \\
\hline 5 & 0.25 & 0.23 \\
\hline 6 & 0.28 & 0.26 \\
\hline
\end{tabular}

Table 5: Estimation error indices obtained for each region from observability Gramian $\left(T_{0}+38\right.$, $\gamma=20 \gamma_{0}$, time $300 \mathrm{~ms}$ ) with synthetic and real observations

conditions are notoriously difficult to handle, and in particular require very smooth surfaces for the sliding to occur without artifacts, which of course is quite challenging for anatomical models obtained from segmented images.

We also note that the estimation at $T_{0}+38$ provides similar contractility values for regions $\sharp 2$ and $\sharp 3$, whereas the corresponding measured infarct proportions significantly differ (64\% versus $20 \%$, respectively). However, the estimated values are consistent with the thickening indices ( $0 \%$ versus $7 \%$, respectively) which show that region $\sharp 3$ is almost akinetic. Beyond the above-mentioned limited accuracy of late enhancement post-processing, this discrepancy may be explained by the fact that - while late enhancement accurately maps the non-viable tissue [28], the akinetic or hypokinetic myocardium in fact extends quite deeply into the infarct border zone [1].

We now discuss some other interpretations of the estimation results over the various stages. At stage $T_{0}+10$, the variations of estimated contractility parameters between the healthy and the infarcted regions are lower than at stage $T_{0}+38$. This is consistent with the fact that at $T_{0}+10$ the ventricle does not yet show any evidence of remodeling in the form of infarcted wall thinning, and that the akinetic parts are mainly located in 2 mid-cavity short axis slices, while the remaining infarcted tissue is only hypokinetic. In addition, whereas there should be an increase of the actual tissue stiffness in the infarct at stage $T_{0}+38$ due to the fibrous tissue, by contrast the stiffness may be reduced at stage $T_{0}+10$ due to tissue edema and the acute inflammation that accompanies the infarction. In our estimation procedure we do not vary the passive stiffness parameter, hence by compensation this should lead to overestimating and underestimating the infarcted tissue contractility at stages $T_{0}+10$ and $T_{0}+38$, respectively. Note that we could have accordingly modified the stiffness moduli before starting the estimation, but this would mean using some a priori knowledge of the infarct location, which is somewhat in contradiction to our purpose. In the future, we will instead consider performing the joint estimation of both contractility and passive tissue stiffness parameters.

At the final stage $\left(T_{0}+38\right)$, however, the large contractility values obtained in regions $\sharp 5$ and $\sharp 6$ remain to be interpreted. Compensatory hypertrophy in the healthy tissue may provide an explanation, and this is corroborated by the very large thickening values measured in these regions, indeed - recall Table 1 . In addition, it should be noted that the animal grew between the acquisition of the baseline stage and the stage $T_{0}+38$, and this factor could also contribute to the increased contractility.

Finally, as regards the estimation based on the 17-segment AHA subdivision, a detailed analysis of the results shows that they are fully consistent with the 6-region estimation. As a result, the estimated values in AHA segments $\downarrow 1, ~ \llbracket 7$ and $\llbracket 13$ - associated with the abovediscussed region $\sharp 1$ in the 6 -region subdivision - are all clearly in excess. Nevertheless, when 
comparing e.g. the estimated values in the AHA segments $₫ 3, \llbracket 9$ and $₫ 14$ - corresponding to the infero-septal region $\sharp 3$ in the 6-region subdivision - the trend shown is in accordance with the late enhancement respective infarct proportions. Therefore, even though we cannot expect excellent quantitative accuracy at this level of detail in the regional subdivision - due to to the limited information contents featured in the boundary segmentation of Cine-MRI data - our preliminary trial with AHA segments shows adequate sensitivities while providing a feasibility study which paves the way for further applications with other types of data, such as tagged-MRI.

\section{Concluding remarks}

In this paper, we proposed a methodology of sequential joint state-parameter data assimilation adapted to a biomechanical heart model with medical imaging measurements, and we demonstrated the application of this approach with actual cardiac Cine-MRI data. As a result, this approach provides an automatic biophysical personalization of the model, which enables further uses for predictive purposes with key perspectives in therapy planning, as e.g. already presented in [45]. In particular, we have obtained much improved simulations when using the estimated parameter values in the model, including in large regions of the model which were not included in the measurements used to perform the estimation procedure, which provides a partial predictivity validation in itself. Furthermore, we were able to localize and quantify myocardial infarction via regional contractility parameters, which shows some valuable potential for diagnosis assistance. We point out that the good correlation of these estimated values with independent data provided by late enhancement imaging - except for one region in which incorrect estimation results have been interpreted in detail - combined with improved simulated kinematics, shows that the estimation procedure does not simply "fit" the simulation with images, and truly enhances predictivity, indeed.

Of course, this work should be primarily seen as a feasibility study, and more extensive validation is required in the perspective of clinical applications. In particular, more cases should be considered, and the predictivity improvements provided by personalized modeling ought to be quantitatively evaluated by resorting to additional independent data, and/or when investigating therapeutic strategies with the model as in [45]. However, with this study we have demonstrated the applicability - and some benefits - of a complete estimation procedure workflow making use of a biophysical heart model with actual clinical data.

Furthermore, with a view to diagnosis assistance, detailed regional contractility maps - in particular with a 17-segment AHA subdivision of the left ventricle, possibly further subdivided into several layers - are an important objective. In this regard, our results indicate that observability conditions intrinsically restrain the information which can be extracted from mere segmented Cine-MR image sequences. Indeed, our preliminary assessments performed with synthetic data have shown that, with a realistic measurement noise level, the accuracy of contractility estimation is good when using the late enhancement information to subdivide the myocardium into two distinct regions, but it is more limited - although a correct sensitivity was obtained - when considering six regions without any prior information on the infarct location. Hence, our preliminary trial with 17-segment contractility estimated from actual data cannot be assessed in a thoroughly quantitative manner, although the results obtained are consistent with the six-region estimation, with some adequate trends observed when comparing with late enhancement imaging. In the future, however, we will also employ tagged MR images, either with directly extracted myocardium displacements [3], or by extending in a straightforward manner the estimator used in this work based on measuring distances between the simulated and observed surfaces with the tag planes [25]. We can conjecture that the level of details accessible with this type of data would be significantly enhanced, and we could also expect to 
benefit from this to estimate some other physical parameters, such as passive tissue stiffness or quantities pertaining to the electrical activation.

\section{A Time discretization schemes}

Regarding the time discretization of the model itself, we choose a second-order mid-point Newmark scheme for the virtual work equation. The time dependent constitutive law is also discretized using a mid-point rule, meaning that the quantities $\frac{1}{2}\left(\left\{e_{c, h}^{n+1}\right\}+\left\{e_{c, h}^{n}\right\}\right), \frac{1}{\Delta t}\left(\left\{e_{c, h}^{n+1}\right\}-\right.$ $\left.\left\{e_{c, h}^{n}\right\}\right), \frac{1}{2}\left(\left\{k_{c, h}^{n+1}\right\}+\left\{k_{c, h}^{n}\right\}\right)$ and $\frac{1}{2}\left(\left\{\tau_{c, h}^{n+1}\right\}+\left\{\tau_{c, h}^{n}\right\}\right)$ are simply substituted for $e_{c}, \dot{e}_{c}, k_{c}$ and $\tau_{c}$ in (9).

We finally obtain the following total discretized formulation

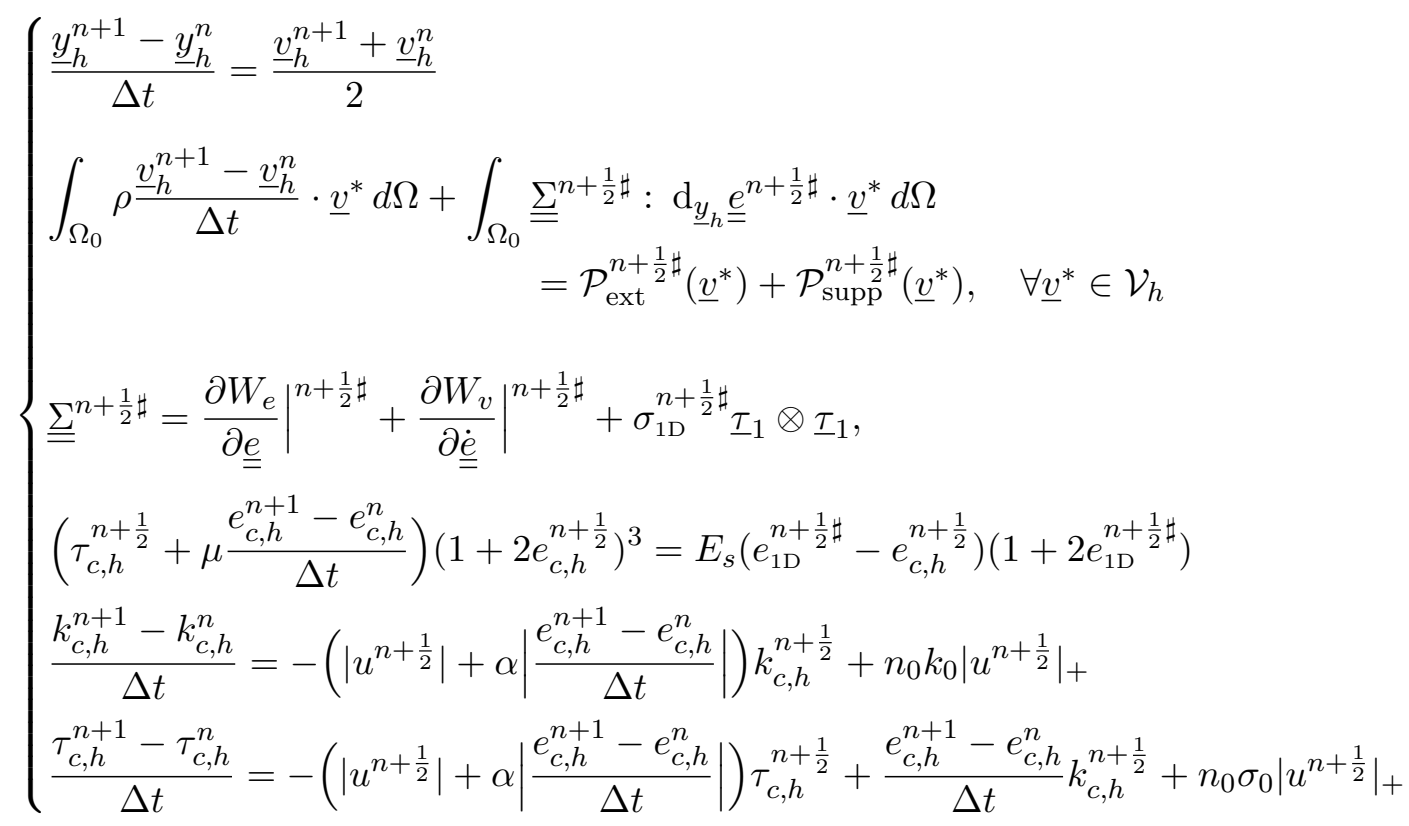

In this system, we use the notation $(\cdot)^{n+\frac{1}{2}}$ to denote either $(\cdot)\left(t_{n+\frac{1}{2}}\right)$ when considering data quantities, or $1 / 2\left((\cdot)^{n+1}+(\cdot)^{n}\right)$ for unknowns. We then resort to the notation $(\cdot)^{n+\frac{1}{2} \sharp}$ for other rules which we now specify. We use

$$
\begin{gathered}
e_{1 \mathrm{D}}^{n+\frac{1}{2} \sharp}=\underline{\tau}_{1} \cdot \underline{\underline{e}}\left(\underline{y}_{h}^{n+\frac{1}{2}}\right) \cdot \underline{\tau}_{1}, \\
\mathrm{~d}_{\underline{y} \underline{\underline{e}}}^{n+\frac{1}{2} \sharp} \cdot \underline{v}^{*}=\frac{1}{2}\left(\underline{\underline{F}}\left(\underline{y} h{ }^{n+\frac{1}{2}}\right)^{T} \cdot \underline{\nabla}_{\underline{\xi}} \underline{v}^{*}+\underline{\nabla}_{\underline{\xi}}^{T} \underline{v}^{*} \cdot \underline{\underline{F}}\left(\underline{y}_{h}^{n+\frac{1}{2}}\right)\right), \\
\left.\frac{\partial W_{e}}{\partial \underline{\underline{e}}}\right|^{n+\frac{1}{2} \sharp}=\frac{\partial W_{e}}{\partial \underline{\underline{e}}}\left(\underline{y}_{h}^{n+\frac{1}{2}}\right),\left.\quad \frac{\partial W_{v}}{\partial \underline{\underline{e}}}\right|^{n+\frac{1}{2} \sharp}=\eta \frac{\underline{e}^{n+1}-\underline{\underline{e}}^{n}}{\Delta t},
\end{gathered}
$$

and the series fiber stress is given by the combined formula

$$
\sigma_{1 \mathrm{D}}^{n+\frac{1}{2} \sharp}=\frac{E_{s} e_{s}^{n+\frac{1}{2}}}{1+2 e_{c, h}^{n+\frac{1}{2}}}=\frac{\sigma_{c, h}^{n+\frac{1}{2} \sharp}}{1+2 e_{s}^{n+\frac{1}{2}}} \text { with } \sigma_{c, h}^{n+\frac{1}{2} \sharp}=\tau_{c, h}^{n+\frac{1}{2}}+\mu \frac{e_{c, h}^{n+1}-e_{c, h}^{n}}{\Delta t} \text {. }
$$

Finally,

$$
\mathcal{P}_{\text {ext }}^{n+\frac{1}{2} \sharp}\left(\underline{v}^{*}\right)=-\sum_{c=l, r} \int_{\Gamma_{\text {endo }}} P_{v, c}^{n+\frac{1}{2}} \underline{n}^{n+\frac{1}{2} \sharp} \cdot\left(\underline{\underline{F}}^{n+\frac{1}{2} \sharp}\right)^{-1} \cdot \underline{v}^{*} J^{n+\frac{1}{2} \sharp} d S,
$$


where $\underline{n}^{n+\frac{1}{2} \sharp}, \underline{\underline{F}}^{n+\frac{1}{2} \sharp}$ and $J^{n+\frac{1}{2} \sharp}$ are directly associated with the mid-point displacements $\underline{y}_{h}^{n+\frac{1}{2}}$, and

$$
\mathcal{P}_{\operatorname{supp}}^{n+\frac{1}{2} \sharp}\left(\underline{v}^{*}\right)=-\int_{\mathcal{B}}\left(a(\underline{\underline{\mathrm{x}}}) \underline{y}_{h}^{n+\frac{1}{2}}+b(\underline{\underline{\mathrm{x}}}) \underline{v}_{h}^{n+\frac{1}{2}}\right) \cdot \underline{v}^{*} d S .
$$

With the state variable $X^{n}=\left(Y^{n} ; V^{n} ;\left\{e_{c, h}^{n}\right\} ;\left\{\tau_{c, h}^{n}\right\} ;\left\{k_{c, h}^{n}\right\}\right)$, System (28) can be rewritten in a compact form using standard finite element operators. We thus have

$$
\left\{\begin{array}{l}
\frac{Y^{n+1}-Y^{n}}{\Delta t}=\frac{V^{n+1}+V^{n}}{2} \\
M \frac{V^{n+1}-V^{n}}{\Delta t}+K^{n+\frac{1}{2} \sharp}\left(X^{n+1}, X^{n}\right)=F^{n+\frac{1}{2} \sharp} \\
\frac{\left\{e_{c, h}^{n+1}\right\}_{i}-\left\{e_{c, h}^{n}\right\}_{i}}{\Delta t}=B_{0}\left(\left\{e_{c, h}^{n+1}\right\}_{i},\left\{e_{c, h}^{n}\right\}_{i},\left\{\tau_{c, h}^{n+\frac{1}{2}}\right\}_{i}, e_{1 \mathrm{D}}^{n+\frac{1}{2} \sharp}\right), \quad \forall \underline{P}_{i} \\
\frac{\left\{k_{c, h}^{n+1}\right\}_{i}-\left\{k_{c, h}^{n}\right\}_{i}}{\Delta t}=B_{1}\left(\left\{e_{c, h}^{n+1}\right\}_{i},\left\{e_{c, h}^{n}\right\}_{i},\left\{k_{c, h}^{n+\frac{1}{2}}\right\}_{i}, u^{n+\frac{1}{2}}\right), \quad \forall \underline{P}_{i} \\
\frac{\left\{\tau_{c, h}^{n+1}\right\}_{i}-\left\{\tau_{c, h}^{n}\right\}_{i}}{\Delta t}=B_{2}\left(\left\{e_{c, h}^{n+1}\right\}_{i},\left\{e_{c, h}^{n}\right\}_{i},\left\{k_{c, h}^{n+\frac{1}{2}}\right\}_{i},\left\{\tau_{c, h}^{n+\frac{1}{2}}\right\}_{i}, u^{n+\frac{1}{2}}\right), \quad \forall \underline{P}_{i}
\end{array}\right.
$$

where $M$ denotes the classical mass matrix, the non-linear operator $K^{n+\frac{1}{2} \sharp}$ is defined by

$$
\forall V^{*}, \quad V^{* T} K^{n+\frac{1}{2} \sharp}\left(X^{n+1}, X^{n}\right)=\int_{\Omega_{0}} \underline{\underline{\Sigma}}_{h}^{n+\frac{1}{2} \sharp}: \mathrm{d}_{\underline{y}} \underline{\underline{e}}_{h}^{n+\frac{1}{2} \sharp} \cdot \underline{v}_{h}^{*} d \Omega,
$$

and $B_{0}, B_{1}$ and $B_{2}$ are directly inferred from $(28)_{4},(28)_{5}$ and $(28)_{6}$, respectively. In practice, the non-linear system (29) is solved by a Newton algorithm, and as described in [43] the internal variables defined at the numerical integration points are computed using a Schur complement procedure.

When the ventricular pressures are not available as data, the valve and Windkessel models are also discretized using a mid-point rule, viz.

$$
\left\{\begin{array}{c}
Q_{c}^{n+\frac{1}{2} \sharp}=-\dot{V}_{c}^{n+\frac{1}{2} \sharp}=-\int_{\partial \mathcal{C}_{c}} \underline{n}^{n+\frac{1}{2}} \cdot\left(\underline{\underline{F}}^{n+\frac{1}{2}}\right)^{-1} \cdot \underline{v}_{h}^{n+\frac{1}{2}} J^{n+\frac{1}{2}} d S \\
=f_{\delta, c}\left(P_{v, c}^{n+\frac{1}{2}}, P_{a r, c}^{n+\frac{1}{2}}, P_{a t, c}\left(t^{n+\frac{1}{2}}\right)\right), \quad c=l, r \\
C_{p, c} \frac{P_{a r, c}^{n+1}-P_{a r, c}^{n}}{\Delta t}+\frac{P_{a r, c}^{n+\frac{1}{2}}-P_{d, c}^{n+\frac{1}{2}}}{R_{p, c}}=Q_{c}^{n+\frac{1}{2} \sharp}, \quad c=l, r \\
C_{d, c} \frac{P_{d, c}^{n+1}-P_{d, c}^{n}}{\Delta t}+\frac{P_{d, c}^{n+\frac{1}{2}}-P_{a r, c}^{n+\frac{1}{2}}}{R_{p, c}}=\frac{P_{v s, c}-P_{d, c}^{n+\frac{1}{2}}}{R_{d, c}}, \quad c=l, r
\end{array}\right.
$$

We then obtain a state vector $X^{n}=\left(Y^{n} ; V^{n} ;\left\{e_{c, h}^{n}\right\} ;\left\{\tau_{c, h}^{n}\right\} ;\left\{k_{c, h}^{n}\right\} ; P_{a r, c=l, r}^{n} ; P_{d, c=l, r}^{n}\right)$ that includes the pressure variables. This system is also solved with a Newton procedure and a Schur complement for the internal variables. Regarding the phase changes that modify $f_{\delta}$, the algorithm is as follows: at the beginning of each time step a phase is chosen and the solution of the system (29)-(30) is computed with the adequate expression of the $f_{\delta}$ function for this phase. After convergence, we check whether the solution is compatible with the selected phase and otherwise change the phase and restart the Newton algorithm. If no phase is compatible, the time step is adapted. 
Finally, regarding the time discretization of the state estimation, we follow exactly [34] and simulate

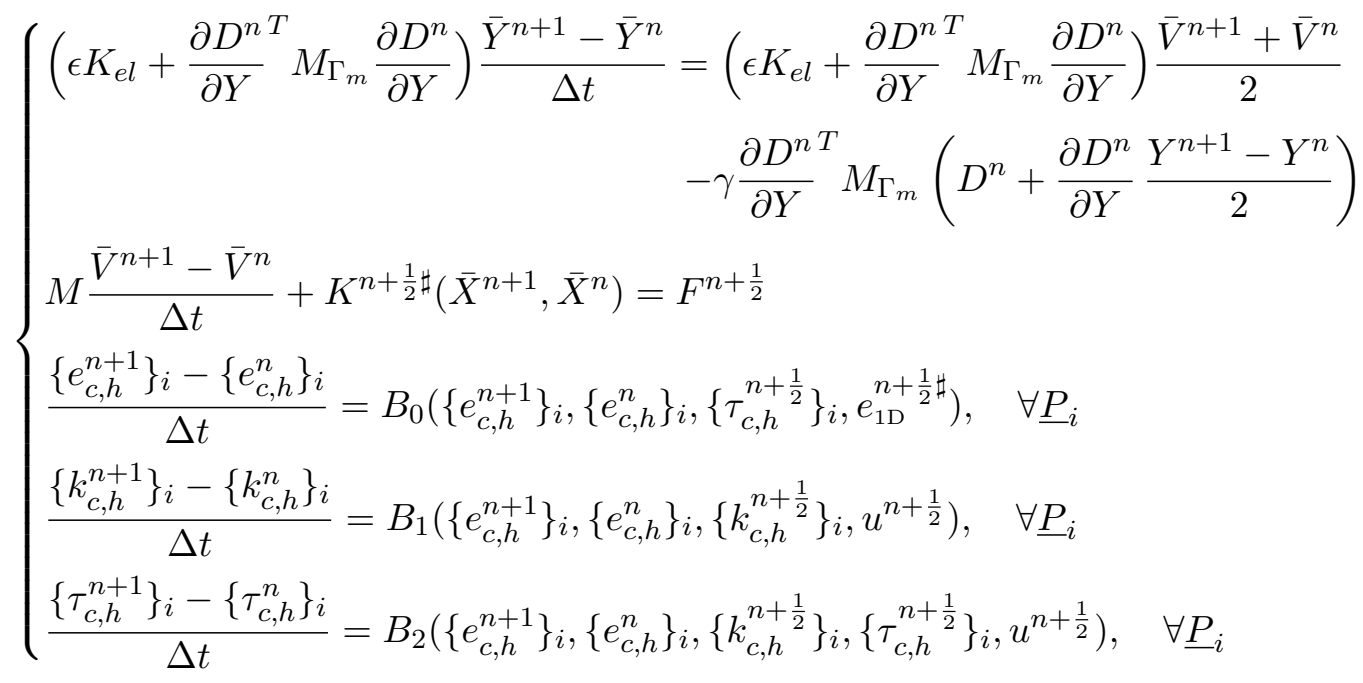

where $D^{n}=D\left(\bar{Y}^{n}, t^{n}\right)$. This problem is solved with a Newton algorithm involving $\bar{Y}^{n+1}$ and $\bar{V}^{n+1}$ with a computational cost close to the original discretization (29) complexity.

\section{References}

[1] H. Ashikaga, S.R. Mickelsen, D.B. Ennis, I. Rodriguez, P. Kellman, H. Wen, and E.R. McVeigh. Electromechanical analysis of infarct border zone in chronic myocardial infarction. Am. J. Physiol. Heart Circ. Physiol., 288:H1099-H1105, 2005.

[2] K.F. Augenstein, B.R. Cowan, I.J. LeGrice, P.M.F. Nielsen, and A.A. Young. Method and apparatus for soft tissue material parameter estimation using tissue tagged magnetic resonance imaging. J Biomech Eng - T ASME, 127(1):148-157, 2005.

[3] L. Axel, A. Montillo, and D. Kim. Tagged magnetic resonance imaging of the heart: a survey. Medical Image Analysis, 9(4):376-393, 2005.

[4] J. Baerentzen and H. Aanaes. Signed distance computation using the angle weighted pseudo-normal. IEEE Trans. Visual. Comput. Graph., 11(3):243-253, 2005.

[5] K.J. Bathe. Finite Element Procedures. Prentice-Hall, 1996.

[6] A. Bensoussan. Filtrage Optimal des Systèmes Linéaires. Dunod, 1971.

[7] J. Bestel, F. Clément, and M. Sorine. A biomechanical model of muscle contraction. In Lectures Notes in Computer Science, volume 2208. Eds W.J. Niessen and M.A. Viergever, Springer-Verlag, 2001.

[8] J. Blum, F.-X. Le Dimet, and I.M. Navon. Data assimilation for geophysical fluids. Computational Methods for the Atmosphere and the Oceans, 14:385-441, 2009.

[9] E. Bogatyrenko and U. Hanebeck. Simultaneous state and parameter estimation for physicsbased tracking of heart surface motion. In Multisensor Fusion and Integration for Intelligent Systems (MFI), 2010 IEEE Conference on, pages 109 - 114, 2010. 
[10] M.D. Cerqueira, N.J. Weissman, and V. Dilsizian et al. Standardized myocardial segmentation and nomenclature for tomographic imaging of the heart: A statement of healthcare professionals from the Cardiac Imaging Comittee of the Council on Clinical Cardiology of the American Heart Association. Circulation, 105:539-542, 2002.

[11] R. Chabiniok, D. Chapelle, P.F. Lesault, A. Rahmouni, and J.F. Deux. Validation of a biomechanical heart model using animal data with acute myocardial infarction. In CI2BM09 - MICCAI Workshop on Cardiovascular Interventional Imaging and Biophysical Modelling, London, UK, 2009.

[12] D. Chapelle, M.A. Fernàndez, J.-F. Gerbeau, P. Moireau, J. Sainte-Marie, and N. Zenzemi. Numerical simulation of the electromechanical activity of the heart. In Proceedings of Functional Imaging and Modeling of the Heart 2009 (FIMH'09), volume 5528 of LNCS, pages 357-365, 2009.

[13] D. Chapelle, P. Le Tallec, P. Moireau, and M. Sorine. An energy-preserving muscle tissue model: formulation and compatible discretizations. International Journal of Multiscale Computational Engineering, 2010. In press.

[14] P.G. Ciarlet and G. Geymonat. Sur les lois de comportement en élasticité non linéaire. C.R.A.S, Série II, 295:423-426, 1982.

[15] K.D. Costa, J.W. Holmes, and A.D. McCulloch. Modeling cardiac mechanical properties in three dimensions. Phil. Trans. R. Soc. Lond. A, 359:1233-50, 2001.

[16] O. Ecabert and N. Smith. euHeart: integrated cardiac care using patient-specific cardiovascular modeling. SPIE Newsroom, 2008.

[17] P.J. Frey and P.L. George. Mesh Generation. Wiley \& Sons, 2008.

[18] S. Göktepe, S. Acharya, J. Wong, and E. Kuhl. Computational modeling of passive myocardium. International Journal for Numerical Methods in Biomedical Engineering, 27:1$12,2011$.

[19] G.A. Holzapfel and R.W. Ogden. Constitutive modelling of passive myocardium: a structurally based framework for material characterization. Phil. Trans. R. Soc. A, 367:3445$3475,2009$.

[20] I. Hoteit, D.T. Pham, and J. Blum. A simplified reduced order Kalman filtering and application to altimetric data assimilation in Tropical Pacific. Journal of Marine Systems, 36(1-2):101-127, 2002.

[21] Z.H. Hu, D. Metaxas, and L. Axel. In vivo strain and stress estimation of the heart left and right ventricles from MRI images. Medical Image Analysis, 7(4):435-444, 2003.

[22] J.D. Humphrey. Cardiovascular Solid Mechanics - Cells Tissues and Organs. Springer, 2002.

[23] P. Hunter et al. A vision and strategy for the virtual physiological human in 2010 and beyond. Phil. Trans. R. Soc. A, 368:2595-2614, 2010.

[24] A.F. Huxley. Muscle structure and theories of contraction. In Progress in Biophysics and Biological Chemistry, volume 7, pages 255-318. Pergamon press, 1957. 
[25] A. Imperiale, R. Chabiniok, P. Moireau, and D. Chapelle. Constitutive parameter estimation methodology using tagged-MRI data. In Proceedings of Functional Imaging and Modeling of the Heart 2011 (FIMH'11). Springer, 2011.

[26] S.J. Julier, J.K. Uhlmann, and H.F. Durrant-Whyte. A new approach for filtering nonlinear systems. In Proceedings of the American Control Conference, volume 3, pages 1628-1632, 1995.

[27] R. Kalman. A new approach to linear filtering and prediction problems. Trans. ASME J. Basic. Eng., 82:35-45, 1960.

[28] R.J. Kim, D.S. Fieno, T.D. Parrish, K. Harris, E.-L. Chen, J.O. Simonetti, J. Bundy, J.P. Finn, F.J. Klocke, and R.M. Judd. Relationship of MRI delayed contrast enhancement to irreversible injury, infarct age, and contractile function. Circulation, 100:1992-2002, 1999.

[29] P. Le Tallec. Numerical methods for nonlinear three-dimensional elasticity. In P. G. Ciarlet and J.-L. Lions, editors, Handbook of Numerical Analysis, volume 3. Elsevier, 1994.

[30] H. Liu and P. Shi. Maximum a posteriori strategy for the simultaneous motion and material property estimation of the heart. IEEE Transactions on Biomedical Engineering, 56(2):378-389, 2009.

[31] T. Mansi, J.-M. Peyrat, M. Sermesant, H. Delingette, J. Blanc, Y. Boudjemline, and N. Ayache. Physically-constrained diffeomorphic demons for the estimation of 3D myocardium strain from Cine-MRI. In Proceedings of Functional Imaging and Modeling of the Heart 2009 (FIMH'09), volume 5528 of LNCS, pages 201-210. Springer, 2009.

[32] P. Moireau and D. Chapelle. Reduced-order Unscented Kalman Filtering with application to parameter identification in large-dimensional systems. ESAIM: COCV, 2010.

[33] P. Moireau, D. Chapelle, and P. Le Tallec. Joint state and parameter estimation for distributed mechanical systems. Computer Methods in Applied Mechanics and Engineering, 197(6-8):659-677, 2008.

[34] P. Moireau, D. Chapelle, and P. Le Tallec. Filtering for distributed mechanical systems using position measurements: perspectives in medical imaging. Inverse Problems, 2009.

[35] P. Moireau, X. Nan, M. Astorino, C.A. Figueroa, D. Chapelle, C.A. Taylor, and J.-F. Gerbeau. External tissue support and fluid-structure simulation in blood flows. Biomechanics and Modeling in Mechanobiology, 2011. In press.

[36] M.P. Nash and P.J. Hunter. Computational mechanics of the heart - from tissue structure to ventricular function. Journal of Elasticity, 61:113-141, 2000.

[37] X. Papademetris, A.J. Sinusas, D.P. Dione, R.T. Constable, and J.S. Duncan. Estimation of 3D left ventricular deformation from medical images using biomechanical models. IEEE Transactions on Medical Imaging, 21(7):786-800, 2002.

[38] J. Peters, O. Ecabert, C. Meyer, R. Kneser, and J. Weese. Optimizing boundary detection via simulated search with applications to multi-modal heart segmentation. Med. Image Anal., 14(70-84), 2010.

[39] D.T. Pham. Stochastic methods for sequential data assimilation in strongly nonlinear systems. Monthly weather review, 129(5):1194-1207, 2001. 
[40] K. Raghavan and A. Yagle. Forward and inverse problems in elasticity imaging of soft tissues. Nuclear Science, IEEE Transactions on, 41(4):1639 - 1648, 1994.

[41] R.S. Rivlin and J.L. Ericksen. Stress-deformation relations for isotropic materials. J. Rational Mech. Anal., 4:323-425, 1955.

[42] F.B. Sachse, R. Frech, C.D. Werner, and O. Dössel. A model based approach to assignment of myocardial fibre orientation. Computers in Cardiology 1999, pages 145-148, 1999.

[43] J. Sainte-Marie, D. Chapelle, R. Cimrman, and M. Sorine. Modeling and estimation of the cardiac electromechanical activity. Computers $\&$ Structures, 84(28):1743-1759, 2006.

[44] J. Schaerer, C. Casta, J. Pousin, and P. Clarysse. A dynamic elastic model for segmentation and tracking of the heart in MR image sequences. Medical Image Analysis, 14:738-749, 2010 .

[45] M. Sermesant, F. Billet, R. Chabiniok, T. Mansi, P. Chinchapatnam, P. Moireau, J.M. Peyrat, K. Rhode, M. Ginks, P. Lambiase, S. Arridge, H. Delingette, M. Sorine, A. Rinaldi, D. Chapelle, R. Razavi, and N. Ayache. Personalised electromechanical model of the heart for the prediction of the acute effects of cardiac resynchronisation therapy. In Proceedings of Functional Imaging and Modeling of the Heart 2009 (FIMH'09), volume 5528 of LNCS, pages 239-248. Springer, 2009.

[46] M. Sermesant, P. Moireau, O. Camara, J. Sainte-Marie, R. Andriantsimiavona, R. Cimrman, D.L. Hill, D. Chapelle, and R. Razavi. Cardiac function estimation from MRI using a heart model and data assimilation: advances and difficulties. Med. Image Anal., 10(4):642-656, 2006.

[47] D. Simon. Optimal State Estimation: Kalman, $H^{\infty}$, and Nonlinear Approaches. WileyInterscience, 2006.

[48] J. Sundnes, G.T. Lines, X. Cai, B.F. Nielsen, K.-A. Mardal, and A. Tveito. Computing the Electrical Activity in the Heart. Springer-Verlag, 2006.

[49] N. Toussaint, T. Mansi, H. Delingette, N. Ayache, and M. Sermesant. An integrated platform for dynamic cardiac simulation and image processing: Application to personalised tetralogy of Fallot simulation. In Proc. Eurographics Workshop on Visual Computing for Biomedicine (VCBM), Delft, The Netherlands, 2008.

[50] N. Toussaint, J.C. Souplet, and P. Fillard. MedINRIA: Medical image navigation and research tool by INRIA. In Proc. of MICCAI'07 Workshop on Interaction in medical image analysis and visualization, 2007.

[51] L. Wang, H. Zhang, K.C.L. Wong, and P. Shi. A reduced-rank square root filtering framework for noninvasive functional imaging of volumetric cardiac electrical activity. In Acoustics, Speech and Signal Processing, 2009. ICASSP 2009. IEEE International Conference on, pages 533-536, 2009.

[52] J. Xi, P. Lamata, J. Lee, P. Moireau, D. Chapelle, and N. Smith. Myocardial transversely isotropic material parameter estimation from in-silico measurements based on reducedorder unscented Kalman filter. J. of the Mechanical Behavior of Biomedical Materials, 2011. In press.

[53] Q. Zhang. Adaptive observer for MIMO linear time varying systems,. IEEE Transactions on Automatic Control, 3:525-529, 2002. 